\title{
Individual colour patches as multicomponent signals
}

\author{
Gregory F. Grether*, Gita R. Kolluru and Karen Nersissian \\ Department of Organismic Biology, Ecology and Evolution, University of California, Los Angeles, 621 Charles E. Young Drive South, \\ Los Angeles, CA 90095-1606, USA
}

(Received 2 December 2002; revised 1 September 2003; accepted 3 September 2003)

\begin{abstract}
Colour patches are complex traits, the components of which may evolve independently through a variety of mechanisms. Although usually treated as simple, two-dimensional characters and classified as either structural or pigmentary, in reality colour patches are complicated, three-dimensional structures that often contain multiple pigment types and structural features. The basic dermal chromatophore unit of fishes, reptiles and amphibians consists of three contiguous cell layers. Xanthophores and erythrophores in the outermost layer contain carotenoid and pteridine pigments that absorb short-wave light; iridophores in the middle layer contain crystalline platelets that reflect light back through the xanthophores; and melanophores in the basal layer contain melanins that absorb light across the spectrum. Changes in any one component of a chromatophore unit can drastically alter the reflectance spectrum produced, and for any given adaptive outcome (e.g. an increase in visibility), there may be multiple biochemical or cellular routes that evolution could take, allowing for divergent responses by different populations or species to similar selection regimes. All of the mechanisms of signal evolution that previously have been applied to single ornaments (including whole colour patches) could potentially be applied to the individual components of colour patches. To reach a complete understanding of colour patch evolution, however, it may be necessary to take an explicitly multi-trait approach. Here, we review multiple trait evolution theory and the basic mechanisms of colour production in fishes, reptiles and amphibians, and use a combination of computer simulations and empirical examples to show how multiple trait evolution theory can be applied to the components of single colour patches. This integrative perspective on animal colouration opens up a host of new questions and hypotheses. We offer specific, testable functional hypotheses for the most common pigmentary (carotenoid, pteridine and melanin) and structural components of vertebrate colour patches.
\end{abstract}

Key words: multiple trait, colour patch, chromatophore, carotenoid, amplifier, mimicry, signal evolution, handicap, indicator.

\section{CONTENTS}

I. Introduction

II. Multiple trait evolution theory

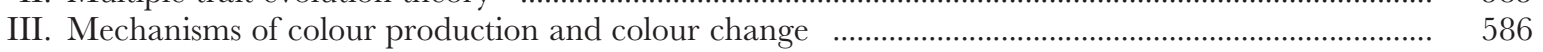

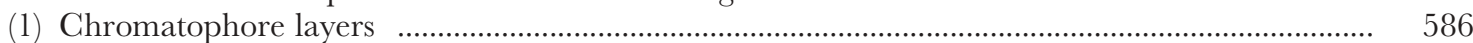

(2) Dermal chromatophore unit ………………………............................................................. 587

(3) Proximate mechanisms of colour change ………………………………............................... 587

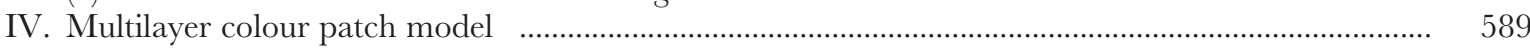

(1) Xanthophores (and erythrophores) (layer 1) ………….................................................................. 589

(2) Iridophores (and leucophores) (layer 2) ……………………………………….......................... 589

(3) Melanophores (layer 3) ……………………......................................................................... 591

(4) Reflective shield of connective tissue (layer 4) ........................................................................... 591

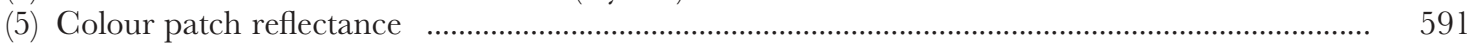

* Address for correspondence: E-mail: ggrether@ucla.edu 
(6) Colour patch radiance ............................................................................................................... 591

(7) Perceived colour contrasts ………….......................................................................................... 591

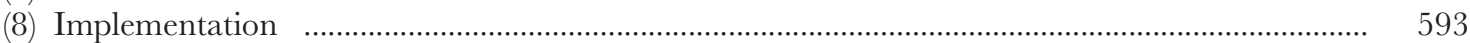

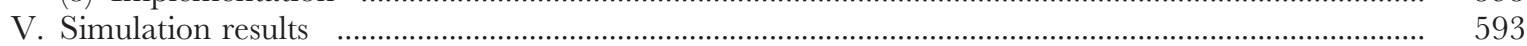

(1) Spectral mimicry and fine-tuning within the xanthophore layer .............................................. 593

(2) The amplifying effects of iridophores …………....................................................................... 594

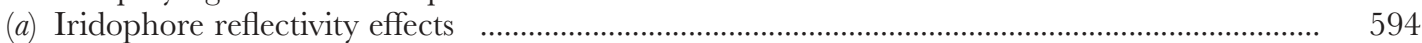

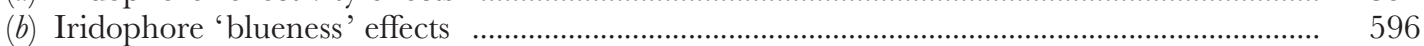

(3) The amplifying and attenuating effects of melanophores _............................................................ 596

(a) The amplifying effect of melanophores on iridophores …………………………................ 597

(b) The effects of melanophores on xanthophores ………………………………….................. 598

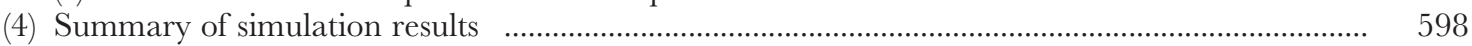

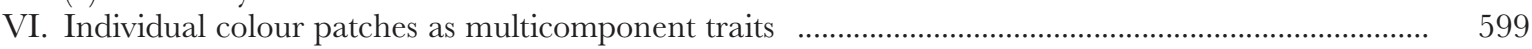

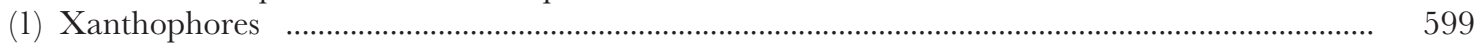

(a) Carotenoids versus pteridines ………………………………………………………...... 599

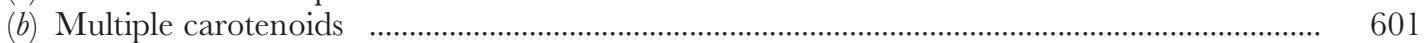

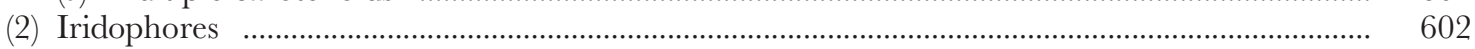

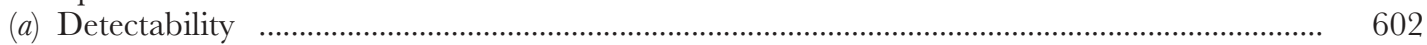

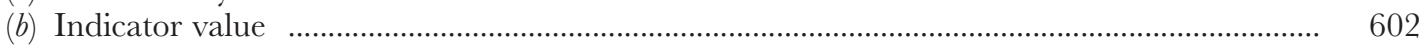

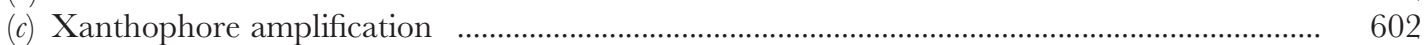

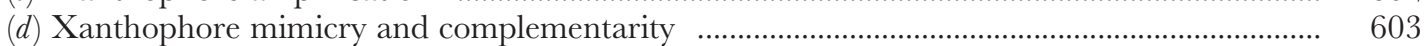

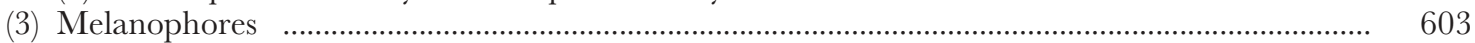

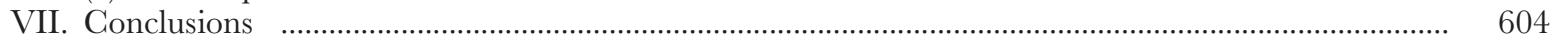

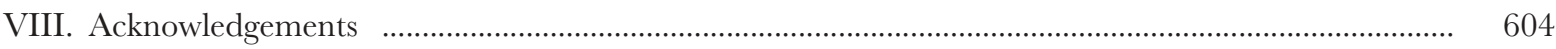

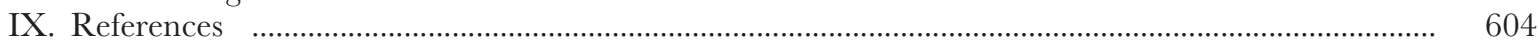

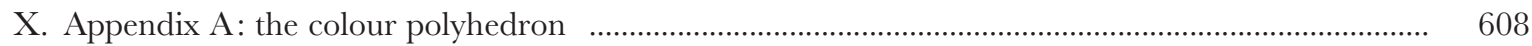

\section{INTRODUGTION}

Vertebrate colour patches are complex traits, the components of which may evolve independently through a variety of mechanisms (Losos, 1985; Thompson \& Moore, 1991; Endler, 1992; Macedonia \& Stamps, 1994; Berglund, Bisazza \& Pilastro, 1996; Macedonia, Brandt \& Clark, 2002). Although usually treated as simple, two-dimensional characters and classified as either structural or pigmentary and, if the latter, by pigment type (e.g. melanin, carotenoid and pteridine), in reality, colour patches are multilayered, three-dimensional structures that often contain multiple pigment types and structural features. The standard dermal chromatophore unit (Bagnara, Taylor \& Hadley, 1968; Bagnara \& Hadley, 1973) of poikilothermic vertebrates consists of three contiguous cell layers (Fig. 1). Xanthophores and erythrophores in the outermost layer contain carotenoid and pteridine pigments that primarily absorb short-wave light; iridophores in the middle layer contain crystalline platelets that reflect light back through the xanthophores; and melanophores in the basal layer contain melanins that absorb light across the spectrum. Changes in any one component of a chromatophore unit can dramatically alter the colour produced. For any given adaptive outcome (e.g. an increase in crypsis), there may be multiple biochemical or cellular routes that evolution could take, allowing for divergent responses by different populations or species to the same selection regimes. Moreover, different colour patch components may evolve in fundamentally different ways.
Our main thesis herein is that the mechanisms of signal evolution that previously have been applied to single ornaments (including whole colour patches) and multiple ornaments (including colour patterns) may also be applied to the individual components of colour patches. This novel perspective on animal colouration opens up a range of new questions. For clarity, we present mainly sexual signalling (mate choice) examples, but the same basic arguments could be applied, for example, to colour patches used in competition for territories or other resources.

The organization of this review is as follows. First, we briefly review multiple trait evolution theory, clarify the relationships between the various models, and explain how our hypothesis differs from previous ideas about composite colour signals (Section II). Next, we review the basic mechanisms of colour production and colour change in the skin of fishes, reptiles and amphibians, and provide examples of colour variants that are understood at a cellular or biochemical level (Section III). The main purpose of the first two sections is to acquaint readers with concepts and terminology that will be used subsequently. In the core of the paper, we derive a general model of a multilayer colour patch (Section IV) and use computer simulations to explore the properties of different colour patch components in a multi-trait context (Section V). Most of these simulations examine how changes of a specified nature in one chromatophore layer are likely to affect the colour perceived and the discriminability of variation in other chromatophore layers. In the final section, we discuss the implications of our findings in the context of multiple 


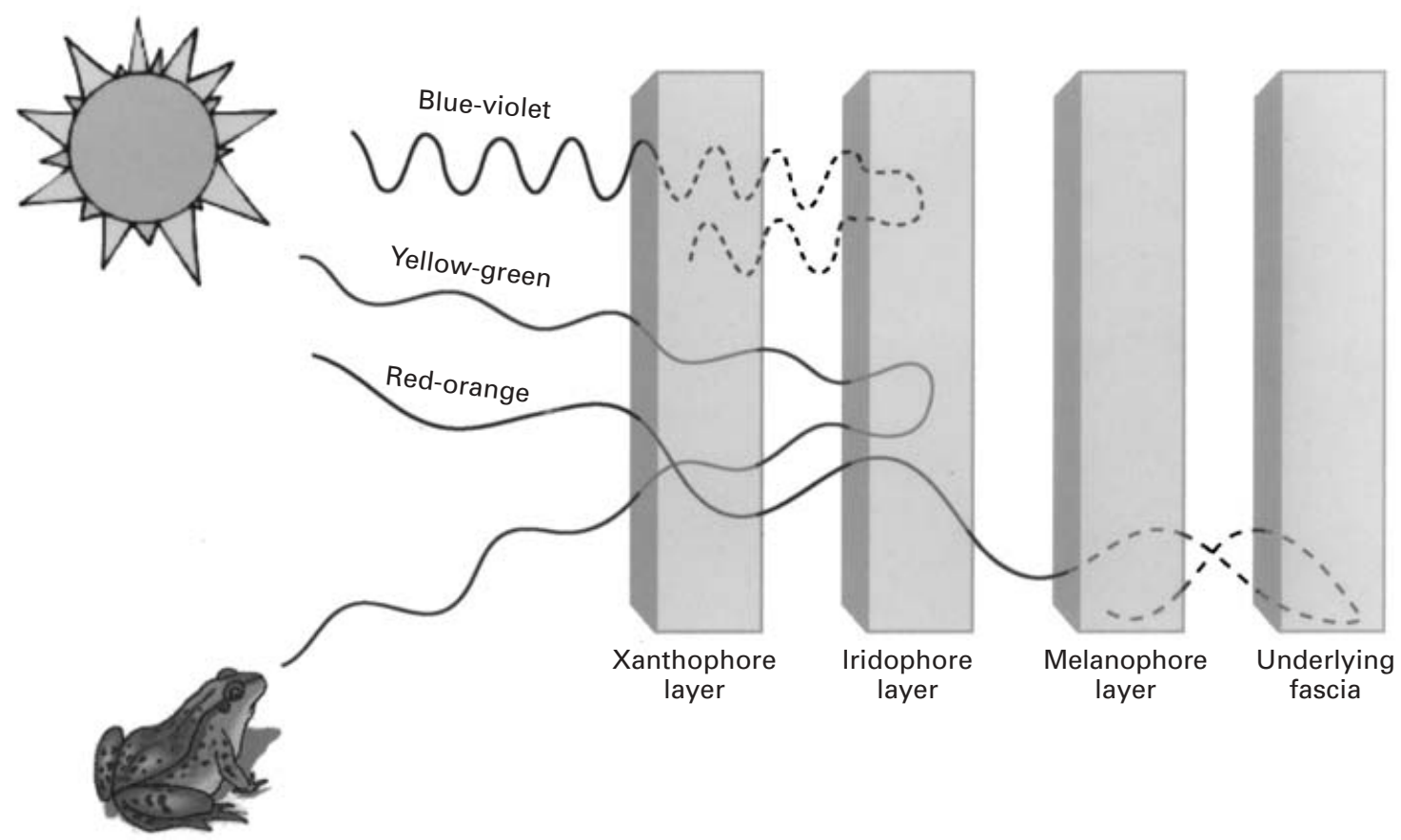

Fig. 1. The dermal chromatophore unit (Bagnara et al., 1968; Bagnara \& Hadley, 1973) of a hypothetical green frog, showing how the xanthophore, iridophore, melanophore and underlying fascia layers interact to determine the overall colour of the animal. Wavy lines depict the paths of light of differing wavelengths through the cell layers. Adapted from Bagnara \& Hadley (1973).

trait evolution theory and suggest avenues for further research (Section VI).

\section{MULTIPLE TRAIT EVOLUTION THEORY}

Multicomponent signals may evolve through a variety of mechanisms operating alone or in combination (Andersson, 1994; Andersson \& Iwasa, 1996; Jennions \& Petrie, 1997; Brooks \& Couldridge, 1999; Rowe \& Guilford, 1999; Andersson et al., 2002). These include runaway selection (Fisher, 1930, 1958), indicator processes (direct and indirect benefits; Darwin, 1871; Zahavi, 1975; Grafen, 1990), sensory exploitation (Ryan \& Keddy-Hector, 1992), status signal selection (Pryke, Andersson \& Lawes, 2001), chaseaway selection (Holland \& Rice, 1998; Brooks \& Couldridge, 1999; but see Rosenthal \& Servedio, 1999), and species recognition mechanisms (Crapon de Caprona \& Ryan, 1990; Pfennig, 1998; Hankinson \& Morris, 2002). Some traits may also function to enhance, mimic, amplify or attenuate other traits (Hasson, 1989, 1990). Of these processes, indicator mechanisms and signal amplification are the most relevant to our examination of individual colour patches as multicomponent traits, and we focus on these below.

Amplifiers and attenuators facilitate or hamper receiver assessment of signalers based on other (indicator) traits (Hasson, 1989, 1990; Table 1). Amplifiers that remain unlinked to signaler quality confer a fitness benefit to high-quality signalers and a cost to low-quality signalers by enhancing the perception of signaler quality (Hasson, 1991). For a 'pure' amplifier to persist, the overall benefits to high-quality signalers must outweigh the costs to low-quality signalers (Hasson, 1991). Alternatively, amplifiers may themselves become linked to quality, in which case they become condition-dependent traits known as amplifying handicaps (Hasson, 1990, 1997). Handicaps are traits that are costly to produce, maintain or possess and whose costs are lower for higher-quality signalers (Hasson, 1997). Amplifying handicaps are costly to produce and also amplify the quality-indicating properties of other traits. For example, white tips and glossy structural colours on bird feathers may be amplifiers because they make feather quality (damage, abrasion) more visible, and handicaps because they increase the probability of feather wear (Fitzpatrick, $1998 a, b$ ). Regardless of the type of amplifier involved, the amplifier process results in multiple-trait displays.

Møller and Pomiankowski (1993) formalized several hypotheses to explain the evolution of multicomponent sexual displays (see also Zuk et al., 1990; Zuk, Ligon \& Thornhill, 1992; Sullivan, 1994; Johnstone, 1996). They distinguished among (1) multiple messages, in which different traits indicate different aspects of signaler quality; (2) redundant signals, which together indicate overall signaler quality; and (3) unreliable signals, which are arbitrary traits not correlated with signaler quality. The outcomes of these and other models of multiple trait evolution rely on the costs to receivers of assessing multiple traits (Iwasa \& Pomiankowski, 
Table 1. Terminology associated with multicomponent trait evolution

\begin{tabular}{|c|c|c|}
\hline Term & Definition & Source \\
\hline Signaler quality & $\begin{array}{l}\text { Variable, hidden attributes of signalers that have the potential } \\
\text { to affect the fitness of receivers or their offspring. }\end{array}$ & \\
\hline Quality indicator & $\begin{array}{l}\text { A trait that honestly signals some aspect of signaler quality. Some } \\
\text { quality indicators are handicaps, traits that are costly to } \\
\text { produce or possess and whose costs are directly responsible } \\
\text { for signal honesty. Handicaps impose a greater relative cost } \\
\text { on low-quality than on high-quality signalers. }\end{array}$ & Zahavi (1975); Hasson (1990) \\
\hline Arbitrary trait & $\begin{array}{l}\text { A sexually selected trait that is not condition-dependent. } \\
\text { For example, a trait that has evolved via Fisherian runaway } \\
\text { selection or via exploitation of pre-existing receiver sensory } \\
\text { biases. }\end{array}$ & $\begin{array}{l}\text { Fisher (1930, 1958); Ryan et al. (1990); } \\
\text { Ryan (1990); Endler (1991 a) }\end{array}$ \\
\hline Amplifier (attenuator) & $\begin{array}{l}\text { A trait not directly preferred by receivers, and not directly } \\
\text { linked to signaler quality, but which increases (decreases) } \\
\text { the ability of receivers to assess signaler quality based } \\
\text { on other traits. Amplifiers are expected to increase the } \\
\text { fitness of high-quality signalers, but decrease the fitness } \\
\text { of low-quality signalers, because of their quality-revealing } \\
\text { properties. }\end{array}$ & Hasson $(1989,1990,1991)$ \\
\hline $\begin{array}{l}\text { Joint costs of mate choice } \\
\text { (joint-choice costs) }\end{array}$ & $\begin{array}{l}\text { Costs associated with choosing based on multiple ornaments, } \\
\text { above and beyond those associated with choosing based on } \\
\text { one ornament. The costs can be equal to or less than the } \\
\text { sum of costs of choosing each trait separately (joint-choice } \\
\text { costs } \leqslant 0 \text { ), or they can be 'super-additive', that is greater } \\
\text { than the sum of the costs of choosing based on each } \\
\text { trait singly. }\end{array}$ & $\begin{array}{l}\text { Pomiankowski \& Iwasa (1993); } \\
\text { Iwasa \& Pomiankowski (1994) }\end{array}$ \\
\hline
\end{tabular}

1994; Sullivan, 1994; Johnstone, 1995; but see Borgia \& Presgraves, 1998). Some authors have suggested that evaluating multiple ornaments may entail lower costs to receivers (Schluter \& Price, 1993; Sullivan, 1994; Rowe, 1999), for instance, because this enables receivers to evaluate signaler attributes more quickly or accurately, but most authors have assumed that the costs of assessing multiple traits are higher than the costs of assessing single traits. The added costs of assessing multiple traits are termed the "joint costs of choice' (Pomiankowski \& Iwasa, 1993; Iwasa \& Pomiankowski, 1994). Only if joint-choice costs are low can multiple ornaments be maintained. Joint-choice costs have been argued to be low for the multiple tail ornaments of male barn swallows (Hirundo rustica) and the multiple colour spots of male guppies (Poecilia reticulata), on the grounds that these traits can be assessed simultaneously by females using a single sensory modality (Brooks \& Couldridge, 1999; Kose \& Møller, 1999).

Previous authors have treated vertebrate colour patterns as composite traits (Fitzpatrick, 1998 b; Brooks \& Couldridge, 1999; McGraw et al., 2002). For example, Fitzpatrick (1998 b) suggested that carotenoid, structural and melanin patches in bird feathers convey multiple messages (general health, developmental stability and feather quality, respectively). By contrast, we are proposing that individual colour patches should be treated as composite traits. We offer this not as an alternative hypothesis but instead as an added layer of complexity that has largely been overlooked (but see Wedekind et al., 1998; Macedonia et al., 2000; Badyaev et al., 2001; Grether, Hudon \& Endler, 2001a). When viewing colour patterns, receivers may, in effect, be evaluating multiple ornaments nested within multiple ornaments.

\section{MEGHANISMS OF GOLOUR PRODUCTION AND GOLOUR GHANGE}

\section{(1) Ghromatophore layers}

The spectacular array of colours seen in the skin of fishes, reptiles and amphibians is due to the presence of pigment cells called chromatophores. These cells include the lightabsorbing melanophores, xanthophores and erythrophores, and the light-reflecting leucophores and iridophores (Fujii, 1993). Classified by colour, xanthophores and erythrophores are yellow to red, leucophores are opaque white, iridophores are iridescent or silvery, and melanophores are brown to black.

Xanthophores and erythrophores contain carotenoids and pteridines, which act as coloured filters, preferentially absorbing short wavelengths (Bagnara \& Hadley, 1973). Carotenoids are hydrophobic molecules that typically absorb visible light primarily in the $400-500 \mathrm{~nm}$ range, producing yellow to red colours. Two major subclasses of carotenoids include the pure hydrocarbon carotenes and the oxygen-containing xanthophylls (Kayser, 1985). Pteridines are hydrophilic compounds that primarily absorb light between 340 and $500 \mathrm{~nm}$ and may appear yellow (xanthopterin and sepiapterin), red (drosopterin or erythropterin), or colourless to the human eye (Kayser, 1985). 
Iridophores and leucophores produce structural colours through some combination of specular reflection, constructive interference and scattering (Herring, 1994). Iridophores contain organized stacks of reflecting crystalline platelets of guanine, hypoxanthine or uric acid (Bagnara, 1966; Fujii, 1993). Light is reflected at the planar surfaces of the platelets and scattered at the edges, but the colour of the reflected light is primarily determined by the thickness, spacing and refractive index of the platelets (Fujii, 1993; Herring, 1994). A given wavelength $(\lambda)$ is reflected most strongly when the optical thickness (thickness $\times$ refractive index) of the platelets and intervening cytoplasmic spaces equals $1 / 4 \lambda$ (Huxley, 1968; Denton \& Land, 1971). Leucophores are dendritic cells containing globular, membranous vesicles filled with cytoplasm, purines or colourless pteridines (Takeuchi, 1976; Fujii, 1993; Oliphant \& Hudon, 1993). Leucophores could potentially produce a wide range of colours through Rayleigh or Mie scattering, depending on the diameter of the vesicles and refractive indices of the substances within them (Herring, 1994). However, most cells identified as leucophores appear white, probably because the vesicles they contain fall within the size range in which wavelength-independent scattering occurs $(>25 \lambda$; Fujii, 1993).

Melanophores contain melanins - highly polymerized compounds synthesized from tyrosine (Bagnara \& Hadley, 1973) - which absorb light throughout the human visible spectrum and into the ultraviolet range, and thus cause darkening of the integument. (Pheomelanins, which are responsible for some of the yellowish and reddish colours seen in mammals, have not been reported in fishes, reptiles or amphibians; Fujii, 1993.)

\section{(2) Dermal chromatophore unit}

Chromatophores are typically arranged in the dermis in three or four contiguous cell layers, collectively referred to as the 'dermal chromatophore unit' (Fig. 1; Bagnara \& Hadley, 1973). Xanthophores (and erythrophores) make up the outermost layer and are found just beneath the basal lamina and associated collagen. The second layer is made up of iridophores and the third layer consists of melanophores. A reflective shield of connective tissue occurs beneath the melanophores in frogs and lizards (Nielsen \& Dyck, 1978; Macedonia et al., 2000), and reflective leucophores have been found below the melanophores in some fishes. Other species lack a reflective shield below the melanophores (reviewed in Obika, 1988; Fujii, 1993).

The dermal chromatophore unit model (Bagnara et al., 1968; Bagnara \& Hadley, 1973) describes how the xanthophore, iridophore and melanophore layers interact to determine the overall reflectance spectrum of a colour patch (see Fig. 1). Carotenoids and pteridines in the xanthophore layer (if present) differentially absorb shorter wavelengths (violet to blue). A fraction of the light that passes through the xanthophores is reflected back out into the environment by platelets in the iridophores. The remaining light is either absorbed by the melanophores or reflected back through the dermal chromatophore unit by the reflective shield. The internal structure of the iridophores affects the colour as well as the brightness of the colour patch. If the reflecting platelets are dispersed and oriented randomly within the cytoplasm, all wavelengths of light will be reflected and the colour patch will appear silver, yellow, orange or red, depending on the amounts and types of pigments present in the xanthophores. If the reflecting platelets are stacked to produce constructive interference in the short-wavelength region, the result will be a structural blue in the absence of xanthophores, or various hues of yellow-green, green or blue-green if xanthophores are present (see Fig. 1). The same three functional elements - a filtering layer (carotenoid), a reflecting layer (keratin) and an absorbing layer (melanin) - are present in a different cellular form in bird feathers (Bagnara \& Hadley, 1973).

\section{(3) Proximate mechanisms of colour change}

Most types of colour change in fishes, reptiles and amphibians, whether within the lifetime of an individual or over evolutionary time, involve changes in one or more components of the dermal chromatophore unit. Rapid (physiological) colour change results from movement of intracellular organelles, while slow (morphological) colour change involves net gains or losses in pigment content (Bagnara \& Hadley, 1973; Fujii, 1993; Painter, 2000; Oshima, 2001). Dispersion of melanosomes causes darkening of the skin, while aggregation of these melanin-containing organelles causes lightening (Bagnara \& Hadley, 1973). Also, migration of melanosomes into the dendritic processes of the melanophores, which extend over the xanthophores, can bring about rapid darkening of a colour patch (Taylor \& Hadley, 1970; Bagnara \& Hadley, 1973; Fujii, 2000). Dispersion of the pigment-containing organelles in xanthophores (xanthosomes) increases absorption of short-wave light by carotenoids and pteridines, thereby intensifying the colour produced, while aggregation of xanthosomes has the reverse effect. Changes in the shape or position of xanthophores relative to iridophores may also contribute to colour change in some species (e.g. Nielsen, 1978).

Dispersion of iridophore platelets and leucosomes increases the total amount of light reflected, while aggregation of these reflective organelles has the opposite effect. Also, as noted above, when iridophore platelets are dispersed in the cytoplasm, they tend to reflect white light, but when they are aggregated into stacks, thin-layer interference effects can drastically alter the spectrum of light reflected (Fujii, 1993). For example, the dark sleeper goby (Odontobutis obscura) changes from yellow to blue as reflecting platelets in the iridophores move from dispersed to aggregated positions (Fujii, Hayashi \& Toyohara, 1991). The blue colour appears to be produced by thin-film interference effects within the platelet stacks, while the yellow colour appears to be caused by pigments in the xanthophore layer and reflection of white light by iridophore platelets. Some of the dramatic colour changes seen in coral reef fishes are caused by coordinated changes in the distance between successive platelets within the aggregated stacks (Fujii, 1993). A similar mechanism of rapid colour change has been reported for the lizard Urosaurus ornatus (Morrison, Sherbrooke \& FrostMason, 1996). 


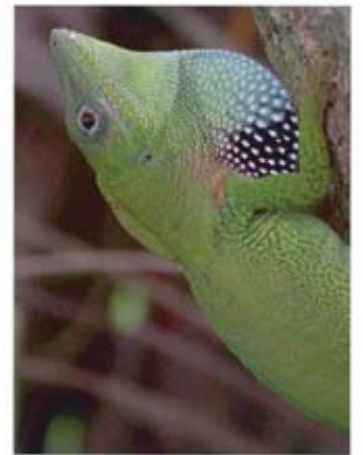

A

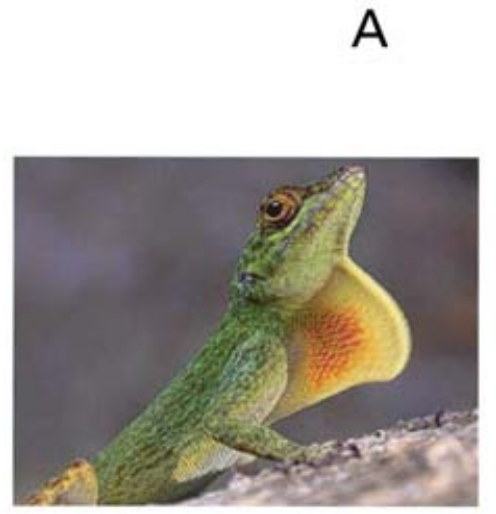

B

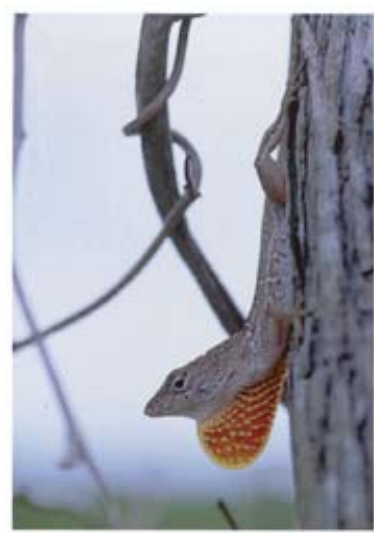

C

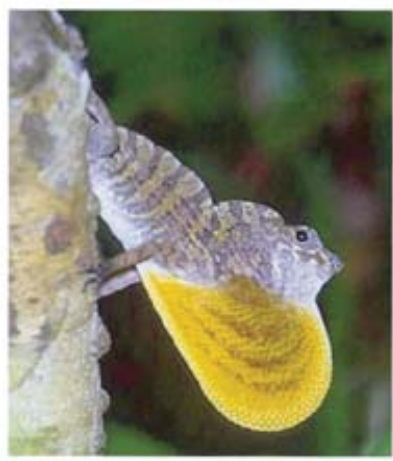

D

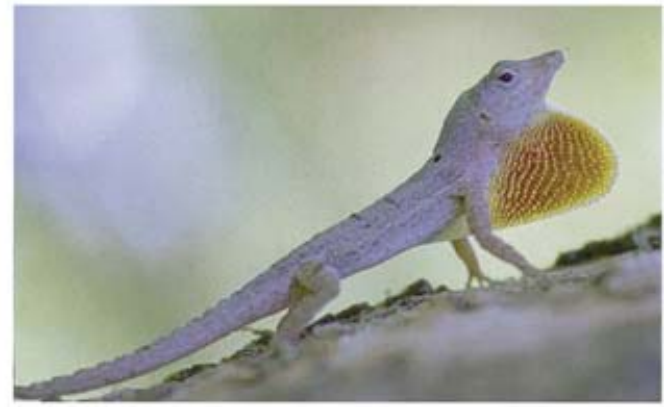

E

Fig. 2. Interspecific variation in dewlap coloration in the Caribbean radiation of Anolis lizards. Photographs show A. chlorocyanus (A) and A. distichus (B) from the Dominican Republic, A. sagrei (C) and A. rubribarbus (D) from Cuba, and A. stratulus (E) and A. pulchellus (F) from Puerto Rico. All photographs (C) Richard Glor.

Tree frogs (Hyla spp.) maintain crypsis against different backgrounds by altering all three chromatophore layers independently (Nielsen, 1978; Nielsen \& Dyck, 1978). Individual Hyla cinerea can assume up to eight recognizably different colours, from black and brown, through various shades of green, to lemon yellow. Based on skin reflectance measurements, Nielsen \& Dyck (1978) inferred that the colour changes are caused by coordinated changes in the dispersion of reflecting platelets, xanthosomes and melanosomes. For example, the transition from olive green to lemon yellow involves dispersion of reflecting platelets and aggregation of melanosomes. Similar mechanisms of colour change have been reported for other species of anurans (Bagnara \& Hadley, 1973; Nielsen, 1978).

Static morph and species differences in colour can also be described in terms of variation in individual components of the dermal chromatophore unit. The three distinct facial colour morphs of the fence lizard, Sceloporus undulatus erythrocheilus, differ mainly with respect to xanthophore pigments and iridophore structure (Morrison, Rand \& Frost-Mason, 1995). In the orange morph, the xanthophores contain red pteridines (drosopterins) and the iridophores produce a complementary orange reflectance spectrum. Likewise, in the yellow morph, the xanthophores contain a yellow compound (riboflavin or sepiapterin) and the iridophores produce a yellow reflectance spectrum. In the melanized morph, the xanthophores are sparse and lack coloured pigments, while the iridophores produce an orange reflectance spectrum, which contributes to the gold tone of this morph. The colour intensity of the yellow and orange morphs changes seasonally, apparently because of changes in the pigment content of the xanthophores. In the non-breeding season, the yellow morph's skin still contains yellow-reflecting iridophores but xanthophore pigments are absent (Morrison et al., 1995).

In the Caribbean radiation of anoline lizards, species differences in dewlap colour have been studied at the phylogenetic, ecological and cellular levels (Fleishman et al., 1997; Persons et al., 1999; Macedonia et al., 2000; Fleishman \& Persons, 2001; Leal \& Fleishman, 2001; Macedonia, 2001; Thorpe, 2002; Fig. 2). Dewlaps are thought to be selected for high visibility in inter- and intrasexual signalling contexts (Fleishman et al., 1997; Persons et al., 1999; Fleishman \& Persons, 2001). To be maximally conspicuous, a colour patch should have a reflectance spectrum matching the ambient irradiance 
spectrum while simultaneously contrasting with the background (Endler, 1993; Endler \& Théry, 1996; Fleishman \& Persons, 2001; Leal \& Fleishman, 2001). Under forest shade, where the ambient light is yellowish-green and the background is predominantly brown and green, a yellow dewlap would be highly conspicuous. Indeed, all three species of Jamaican anoles with yellow dewlaps inhabit forest shade (Macedonia et al., 2000). The dewlaps of these species are yellow due to the presence of the carotenoid zeaxanthin and the absence of red pteridines in the xanthophore layer. By contrast, the four species of Jamaican anoles that possess orange or red dewlaps, due to the presence of red pteridines (drosopterins) in the xanthophore layer, are found in habitats where the ambient light spectrum is relatively rich in long wavelengths (forest gaps and open areas).

One heliophilic Jamaican anole with an orange dewlap (Anolis grahami) is thought to be the ancestor of a shadedwelling species with a deep blue, ultraviolet (UV)-reflective dewlap (A. conspersus) on Grand Cayman. Under the evolutionary scenario proposed by Macedonia (2001), A. grahami colonized Grand Cayman when this island was still arid and unforested, and would therefore have been forced to seek the shade of low shrubs to avoid evaporative water loss. In this woodland/desert shade environment, where shortwave skylight dominates the spectrum (Endler, 1993), a blue, UV-reflective dewlap would be highly conspicuous. The colour shift was achieved through a loss of carotenoids and drosopterins in the xanthophores and the addition of a melanin layer beneath the melanophores in the hypodermis. The loss of xanthophore pigments reduces the absorption of short-wave light and the extra layer of melanin further augments the blue colour by preventing long-wave light that passes through the iridophores from being reflected back by the connective tissue (Macedonia, 2001).

\section{MULTILAYER GOLOUR PATGH MODEL}

Although the basic properties of the chromatophore layers and the roles they play in colour production are well understood (see previous section), it was necessary to construct a model to understand how these properties interact in a signalling context. For example, would a highly reflective iridophore layer make it easier, or more difficult, for females to discriminate between males based on the carotenoid content of their xanthophore layers? Rephrasing the question in signal evolution terms, is iridophore brightness an amplifier or an attenuator of carotenoid colouration? This question is pertinent to a host of species in which carotenoids appear to be indicators of male quality (reviewed in Andersson, 1994). Our simulations show that the answer depends on both viewing conditions and the visual processing mechanisms employed by the receiver (see Section V.2).

The multilayer colour patch model that we derive below is essentially a mathematical representation of the dermal chromatophore unit model of Bagnara et al. (1968) and Bagnara \& Hadley (1973), with some modifications to take into account our current understanding of how chromatophores work (reviewed in Fujii, 1993; Herring, 1994). Although the model is intended to apply to all species with dermal chromatophores, it was necessary to use specific parameter values, and to make assumptions about ambient lighting conditions and the visual system of the receiver, before the features of the model could be explored. We chose to parameterize the model for the iridophores, xanthophore pigments and visual (cone) pigments of guppies (Poecilia reticulata), one of the few species for which such data were available. These initial parameter estimates only provided a starting point, however. In computer simulations, we varied the properties of all chromatophore layers and examined the sensitivity of the results to ambient lighting conditions and visual processing mechanisms. Our findings pertain to taxa with dermal chromatophores generally, and may also be extended, by analogy, to birds.

We now build the model, one chromatophore layer at a time, from the outermost layer to the innermost layer, and derive an equation for calculating the reflectance spectrum of the colour patch. Next we take into account ambient lighting and transmission properties of the medium to derive an equation for patch radiance, which is essentially the amount light of each wavelength reaching the receiver's eyes. Finally, we convert colour patch radiances into estimates of perceived colour contrast.

\section{(1) Xanthophores (and erythrophores) (layer 1)}

Chromatophores of this type can contain carotenoids, pteridines or both types of pigments (in separate cytoplasmic vesicles; Fujii, 1993). Absorbance of light by this layer was calculated from:

$$
A_{1}(\lambda)=\sum c_{i} A_{1 i}(\lambda)
$$

where $A_{1 i}(\lambda)$ is the normalized absorbance of pigment $i$ at wavelength $\lambda$, and $c_{i}$ is the concentration coefficient $(\geqslant 0)$ of pigment $i$. Transmission through this layer is given by:

$$
\mathcal{T}_{1}(\lambda)=10^{-A_{1}(\lambda)}
$$

where $T_{1}(\lambda)$ is the proportion of light transmitted through this layer at wavelength $\lambda$. We modelled the effects of varying the relative contributions of a 'yellow' carotenoid (tunaxanthin; $\lambda_{\max } 440 \mathrm{~nm}$ ), an 'orange-red' carotenoid (astaxanthin; $\lambda_{\max } 467 \mathrm{~nm}$ ) and a 'red' pteridine (drosopterin; $\lambda_{\max } 477 \mathrm{~nm}$ ). Absorbance spectra were obtained from pigment extracts (Hudon, Grether \& Millie, in press) and normalized by dividing by the maximum absorbance (Fig. 3A).

\section{(2) Iridophores (and leucophores) (layer 2)}

Iridophores are complex, multilayered structures. Changes in the number, size, shape, orientation and spacing of the reflecting platelets contained within these cells can affect the spectrum of light reflected in numerous ways (reviewed in Fujii, 1993; Herring, 1994). We modelled two specific 

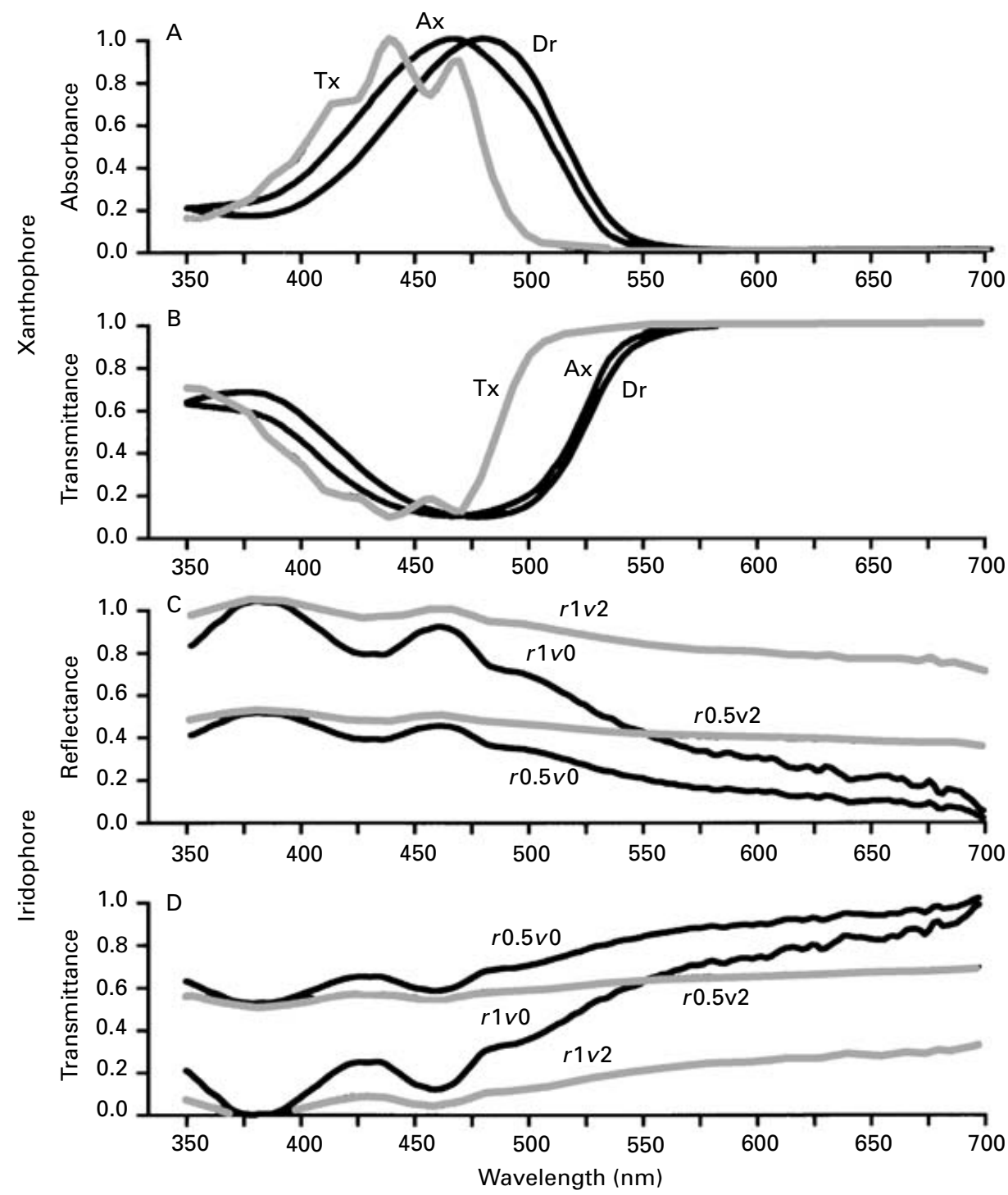

Fig. 3. Representative spectra used in the multilayer colour patch model. (A) Normalized absorbance spectra of the pure xanthophore pigments, tunaxanthin (Tx), astaxanthin (Ax), and drosopterin (Dr), (B) simulated transmittance spectra of the pure xanthophore pigments (from equation 2), (C) reflectance spectra of the iridophore layer for two values of the dilution $(v)$ and reflectivity $(r)$ factors (from equation 3). The spectrum labelled $r 1 v 0$ is the average reflectance spectrum of blue colour spots on male guppies. (D) Simulated transmittance spectra for reflectance spectra shown in (C) (from equation 4). In the model, incident light is assumed to be absorbed by the xanthophore pigments (A) or transmitted to the iridophores $(\mathrm{B})$ and then either reflected back through the xanthophores $(\mathrm{C})$ or transmitted to the melanophore layer $(\mathrm{D})$.

types of changes in the reflective properties of this layer while holding peak wavelength constant. Reflectance from this layer was calculated as:

$$
R_{2}(\lambda)=r(R(\lambda)+v) / \max (R(\lambda)+v),
$$

where $r$ is a reflectivity factor $(0-1), v$ is a dilution factor $(\geqslant 0)$, and $R(\lambda)$ is the reflectance $(0-1)$ at wavelength $\lambda$ of a structural 'blue' reflectance spectrum. The value of $r$ affects total reflectance (brightness) without affecting wavelength composition (spectral shape). The value of $v$ affects the degree to which short wavelength reflectance is diluted by long wavelength reflectance. Dividing by the maximum value of $R(\lambda)+v$ re-scales the spectrum to maintain a maximum reflectance of 1 . To human eyes, as $v$ increases the iridophore spectrum would gradually shift from deep blue to sky blue and eventually appear white. Instead of simulating the structural blue reflectance spectrum itself (which would require numerous assumptions about subcellular structures; Huxley, 1968; Fujii et al., 1991), we used the average reflectance spectrum of the blue spots of male guppies from a single stream in Trinidad (Fig. 3G). At the intracellular level, an increase in $v$ could be produced by increasing the number of dispersed or randomly oriented (as opposed to 
regularly stacked) reflecting platelets, or by increasing the amount of white-light-scattering structures, such as collagen fibrils (see Fujii et al., 1991, p. 469). An increase in $r$ could be produced by increasing the intracellular density of white-light-reflecting organelles or the thickness of the iridophore cell layer. Transmission through this layer is given by:

$$
T_{2}(\lambda)=1-R_{2}(\lambda)
$$

where $T_{2}(\lambda)$ is the proportion of light transmitted through this layer at wavelength $\lambda$.

\section{(3) Melanophores (layer 3)}

Absorbance by the melanophore layer is given by $d A_{3}(\lambda)$ where $d$ represents the density of melanin $(\geqslant 0)$ and $A_{3}(\lambda)$ is the normalized absorbance function for melanin at wavelength $\lambda$. Transmission through this layer is given by:

$$
\mathcal{T}_{3}(\lambda)=10^{-d A_{3}} \text {. }
$$

Biologically, an increase in $d$ would be equivalent to increasing the intracellular density or dispersion of melanosomes (melanin-containing vesicles) or the thickness of the melanophore cell layer. Different types of melanin have different spectral properties (varying shades of brown). For the purposes of our simulations, however, we simplified matters by treating melanin as spectrally neutral (absorbing evenly across the spectrum).

\section{(4) Reflective shield of connective tissue (layer 4)}

Some species possess a reflective shield of connective tissue below the melanophores, while others do not (Fujii, 1993). For simplicity, we treat this layer as spectrally neutral and $100 \%$ reflective (white) if present [all $R_{4}(\lambda)=1$ ] and $0 \%$ reflective if absent [all $R_{4}(\lambda)=0$ ].

\section{(5) Colour patch reflectance}

Reflectance from the surface of the colour patch, $R_{\text {total }}(\lambda)$, is given from the sum of two separate light paths: (a) $T_{1}(\lambda)^{2} R_{2}(\lambda)$ : light reflected from the iridophore layer that passes twice through the xanthophore layer; (b) $T_{1}(\lambda)^{2}$ $T_{2}(\lambda)^{2} T_{3}(\lambda)^{2} R_{4}(\lambda)$ : light reflected off the reflective shield that passes twice through the xanthophore, iridophore and melanophore layers.

This model contains a number of simplifications but serves the intended purpose of enabling us to examine the influence of certain chromatophore properties on colour patch reflectance. The model could easily be modified to examine the influence of other chromatophore properties, such as the shape of the melanophore absorption spectrum.

\section{(6) Colour patch radiance}

To calculate the spectrum of light reaching the receiver's eyes, the ambient irradiance spectrum illuminating the colour patch, $I(\lambda)$, must be specified. In our simulations, we examined the results for five different irradiance spectra: a

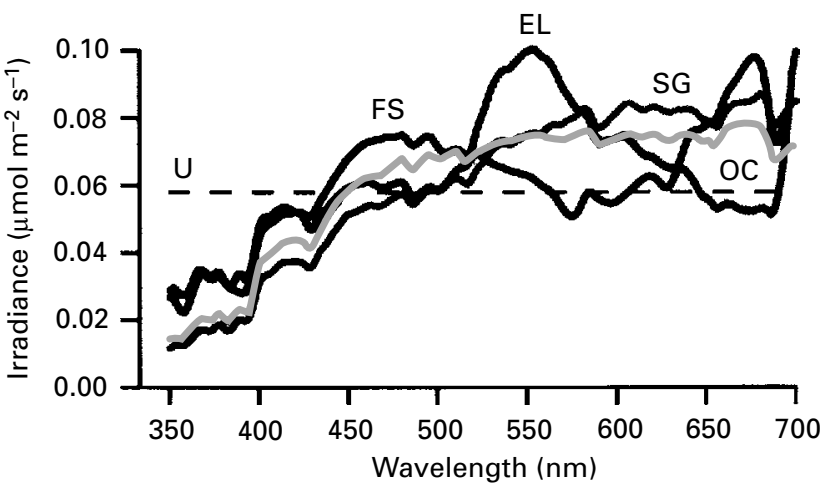

Fig. 4. Ambient light spectra used in the computer simulations: uniform (U), forest shade (FS), early/late (EL), small gap (SG), and open/cloudy (OG). Each spectrum is the average of multiple absolute irradiance measurements taken by J. A. Endler and standardized to a total irradiance of $10 \mu \mathrm{mol} \mathrm{m}^{-2} \mathrm{~s}^{-1}$ (see Endler, 1993). The EL spectrum is the average of the three early/late spectra presented in Endler (1993).

uniform (white) spectrum and natural spectra for four different forest light environments: open/cloudy, small gap, early/late and forest shade (Endler, 1993; Fig. 4). The amount of light of a particular wavelength $(\lambda)$ reaching the receiver's eyes from the simulated colour patch is given by:

$$
Q(\lambda)=I(\lambda) R_{\text {total }}(\lambda) T_{m}(\lambda)
$$

where $Q(\lambda)$ is the patch radiance, in units proportional to photon flux, and $T_{m}(\lambda)$ is the proportion of light of wavelength $\lambda$ that is transmitted through the medium (air or water) to the receiver's eyes. We set $T(\lambda)=1$ for all wavelengths and also assumed an absence of veiling light, which approximates the situation in clear air or water under close viewing conditions (see Endler, 1991 b).

\section{(7) Perceived colour contrasts}

Colour is a property not of objects but of visual systems, so it was necessary to incorporate assumptions about colour vision into our model. The perception of colour is based on contrasts between photoreceptor (cone) outputs, but there is no universally accepted method for calculating colour contrasts (perceived differences in colour), even for extremely well-studied species like humans and honeybees. Some colour discrimination experimental results are best explained by some 'colour space' models and other results are best explained by other models (Chittka, 1999; Vorobyev, 1999; Kelber, Vorobyev \& Osorio, 2003; and references therein). Indeed, in the course of our simulations, we discovered that different colour space models sometimes agreed and other times yielded conflicting results with respect to the phenomena of interest. Our goal here is to illustrate how changes in one chromatophore layer could potentially affect the colour produced and the discriminability of variation in other chromatophore layers, not to 


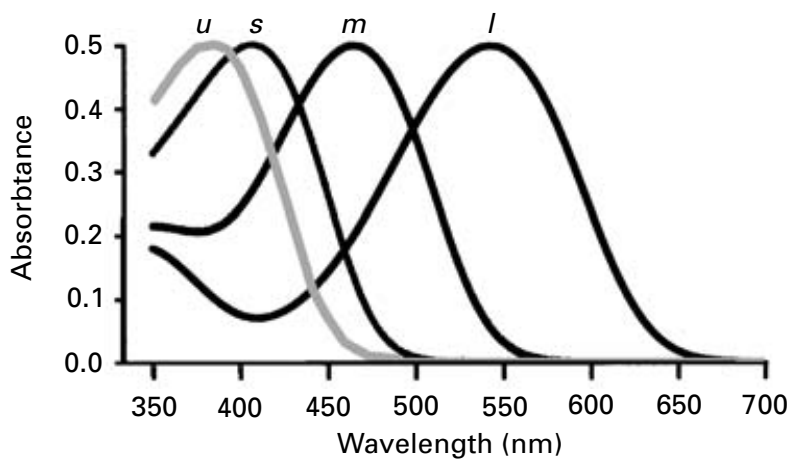

Fig. 5. Spectral sensitivity (absorbtance) functions of guppy photoreceptor cones, estimated from the published maximum absorbance $\left(\lambda_{\max }\right)$ values of $389 \mathrm{~nm}$ ( $u$, ultraviolet), $410 \mathrm{~nm}$ ( $s$, short wavelength), $465 \mathrm{~nm}$ ( $m$, medium wavelength), and $543 \mathrm{~nm}$ ( $l$, long wavelength) (Archer \& Lythgoe, 1990) using the method of Stavenga et al. (1993) and an optical sensitivity of 0.3 (based on Table 11.1 in Nicol \& Somiya, 1989).

compare and evaluate models of colour vision, but we note where the models that we examined gave conflicting results.

Most models of colour vision start with photoreceptor (cone) quantum catch estimates, which can be calculated as:

$$
P_{j}=\int_{\lambda} Q(\lambda) S_{j}(\lambda) d \lambda,
$$

where $Q(\lambda)$ is defined as above, $S_{j}(\lambda)$ is the spectral sensitivity function of photoreceptor class $j, P_{j}$ is in units proportional to photons, and the integration is done over all wavelengths to which the photoreceptor responds (Endler, 1991 b; Chittka, 1992; Vorobyev et al., 1998). In our simulations, we used the spectral sensitivity functions of guppy cones (Fig. 5).

Endler $(1991 b)$ derived a simple method for estimating colour contrasts based on relative cone inputs. Contrasts between pairs of cones are calculated as:

$$
D_{j k}=\left(w_{j} P_{j}-w_{k} P_{k}\right) /\left(w_{j} P_{j}+w_{k} P_{k}\right),
$$

where $w_{j}$ and $w_{k}$ are constants corresponding to how the visual system weighs the output of the different cone classes, and $P_{j}$ and $P_{k}$ are the quantum catch estimates for cone classes $j$ and $k$, respectively. The maximum absolute value of $D_{j k}$ over all cone pairs, or $D_{\max }$, provides an estimate of chroma - the perceived colour difference between a colour patch and an achromatic (white, grey or black) object on a $0-1$ scale. The chroma difference between two colour patches can be calculated as the difference between their respective values of $D_{\max }$. $D_{\max }$ values are relative because the absolute difference between cone classes in photon capture is removed by dividing by the sum of the weighted cone catches. No specific cone opponency assumptions are required by this method, other than that the visual system is capable of comparing all possible pairs of cone outputs. The method can be applied to any species with multiple

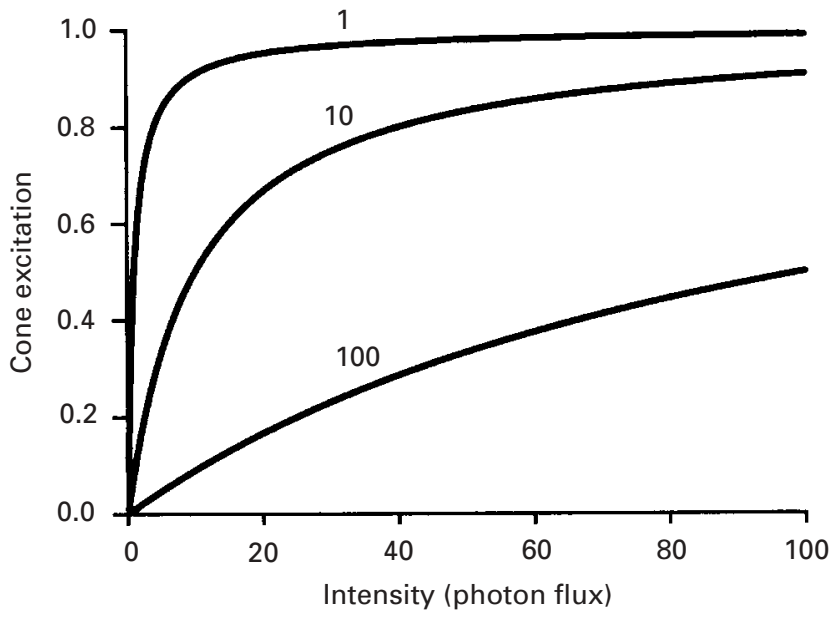

Fig. 6. Hyperbolic relationship between cone excitation and photon capture. The vertical axis represents cone excitation (receptor voltage $V$ ) as a proportion of the maximum $\left(V_{\max }\right)$. The scale of the horizontal axis is arbitrary but proportional to photon flux. The three curves represent three different adaptation light intensities: 1, 10 and 100 on the same scale as the intensity axis. Thus, when the viewer's eyes are adapted to an ambient light intensity of 10 , an object producing an intensity of 10 produces half of the maximal cone response (0.5), while an object producing an intensity of 100 produces a nearly saturated response $(>0.9)$ (based on Laughlin, 1981).

cone types. The w values for guppy cones are unknown and so were set to 1.0 in our simulations, following Endler (1991 b).

Chittka's (1992) 'colour hexagon' model accounts for the non-linear (hyperbolic) relationship between cone excitation and photon capture (Fig. 6), and provides a method for calculating perceived colour contrasts based on absolute cone excitations. Cone excitations are calculated from:

$$
E_{j}=\left(K_{j} P_{j}\right)^{n} /\left(\left(K_{j} P_{j}\right)^{n}+1\right),
$$

where $E_{j}$ is the excitation level of cone class $j$, expressed as a proportion of the maximum receptor voltage, and $K_{j}$ is the reciprocal of the photon catch required to produce half-maximal excitation in cones of class $j$. The value of $K_{j}$ is assumed to depend on the light spectrum to which the receiver's eyes are adapted, such that all cones reach halfmaximal excitation when the receiver's eyes are illuminated by the adaptation light (Laughlin, 1981). The exponents $n$ are photoreceptor- and species-specific, and generally unknown; following Chittka (1992), we set these exponents to 1.0 in our simulations (but see Vorobyev, 1999). The colour hexagon is a two-dimensional colour space diagram in which the $E_{j}$ for three cone classes are represented by vectors oriented 120 degrees from each other. By definition, all achromatic spectra map to the centre of the diagram, because all three cone excitation vectors are equal and thus cancel each other out. The Euclidean distance $Z_{(1-2)}$ between any two points in this colour space provides a generalized measure of perceived colour contrast. All possible cone opponencies are 
represented in the model. Depending on the actual opponency mechanisms and cone weighting factors used by the animal, the generalized model may over- or underestimate perceived colour contrasts. For our purposes, however, the generalized version of the model is preferable to a species-specific version.

Although designed for trichromatic animals (species with three cone types), Chittka's (1992) model can readily be extended to dichromats, in which case the colour space is a line with two vectors oriented 180 degrees from each other. In Appendix A, we derive the tetrachromatic version of the model, which consists of four vectors in three-dimensional space separated from each other by $109.47^{\circ}$ angles. The resulting colour space - a polyhedron with 14 vertices - is of limited use as a colour opponency diagram. Mathematically, however, the tetrachromatic model has the same properties as the trichromatic model and can be used to estimate perceived colour contrasts.

Plotting cone excitations in colour space requires that they first be converted to $x, y$ and $z$ coordinates as follows: For dichromats :

$$
\begin{aligned}
& x=E_{l}-E_{s}, \\
& y=0, \\
& z=0 .
\end{aligned}
$$

For trichromats:

$$
\begin{aligned}
& x=0.866\left(E_{l}-E_{s}\right), \\
& y=E_{m}-0.5\left(E_{s}-E_{l}\right), \\
& z=0 .
\end{aligned}
$$

For tetrachromats:

$$
\begin{aligned}
& x=0.943 E_{m}-0.471\left(E_{s}+E_{u}\right), \\
& y=E_{l}-0.333\left(E_{m}+E_{l}+E_{u}\right), \\
& z=0.816\left(E_{u}-E_{l}\right) .
\end{aligned}
$$

where the subscripts $u, s, m$ and $l$ identify the wavelength class of guppy cones used in our simulations (see Fig. 5). The distance $Z_{(1-2)}$ between any two colours (points in colour space) can be calculated from:

$$
z_{(1-2)}^{2}=\left(x_{1}-x_{2}\right)^{2}+\left(y_{1}-y_{2}\right)^{2}+\left(z_{1}-z_{2}\right)^{2} .
$$

\section{(8) Implementation}

The model described above was implemented on a Macintosh G4 computer (Apple Computer Inc., Cupertino, California, USA) using spreadsheet formulae and Visual Basic macros in Excel 98 (Microsoft Corporation, Seattle, Washington, USA).

\section{SIMULATION RESULTS}

\section{(1) Spectral mimicry and fine-tuning within the xanthophore layer}

The existence of multiple pigment types in the xanthophore layer raises some interesting possibilities for signal evolution. There are several reasons to think that carotenoids are more costly for animals to display in the integument than pteridines (see Section VI). Briefly, carotenoids must be obtained from the diet and may therefore be more difficult to acquire than pteridines, which are endogenously produced. Moreover, a potential trade-off exists between the health-related benefits of carotenoids and their signalling benefits because, for example, the same molecule cannot simultaneously be used for mate attraction and immune system function. Pteridines, by contrast, have no known links to health. The spectral similarity of these two pigment classes suggests that pteridines could be used as a cheap substitute for carotenoids, perhaps undetectably (Grether et al., $2001 a$; Hudon et al., in press).

Pteridines and carotenoids have different-shaped absorption spectra, so no one pteridine is likely to provide a close match to a particular carotenoid. However, it might be possible to achieve a close match by combining pteridines with other carotenoids, the benefit being a reduction in the total amount of carotenoids required to produce a particular colour. Our simulations were designed to illustrate the latter possibility as well as the more general principle that different xanthophore pigments can be combined for fine-tuning the perceived colour.

We simulated the effects of varying the relative amounts of tunaxanthin and drosopterin in the xanthophore layer and compared the results to a simulated colour patch containing only astaxanthin. Tunaxanthin (a carotenoid) and drosopterin (a pteridine) are the main pigments in the xanthophores of guppies; astaxanthin is a common ketocarotenoid found, for example, in crustaceans and salmonid fish (Matsuno, 2001). To human eyes, tunaxanthin is yellow, astaxanthin is orange-red, and drosopterin is red. Drosopterin itself comes close to matching the spectral properties of astaxanthin (absorption and transmission spectra are shown in Fig. 3). The main purpose of our simulations was to determine whether astaxanthin could be matched more exactly by a combination of drosopterin and tunaxanthin.

Our simulations show that the properties of astaxanthin can indeed be mimicked by combining drosopterin and tunaxanthin, while holding the total amount of pigment in the xanthophore layer constant. As shown in Fig. 7, the reflectance spectrum of a pure astaxanthin colour patch cannot be matched precisely by any combination of tunaxanthin and drosopterin. It is possible, however, for different reflectance spectra to generate the same pattern of cone stimulation and therefore appear identical to the receiver. The cone excitation model suggests that a $40 \%$ tunaxanthin, $60 \%$ drosopterin colour patch would look nearly identical to a colour patch containing only astaxanthin, when viewed through the eyes of guppies 


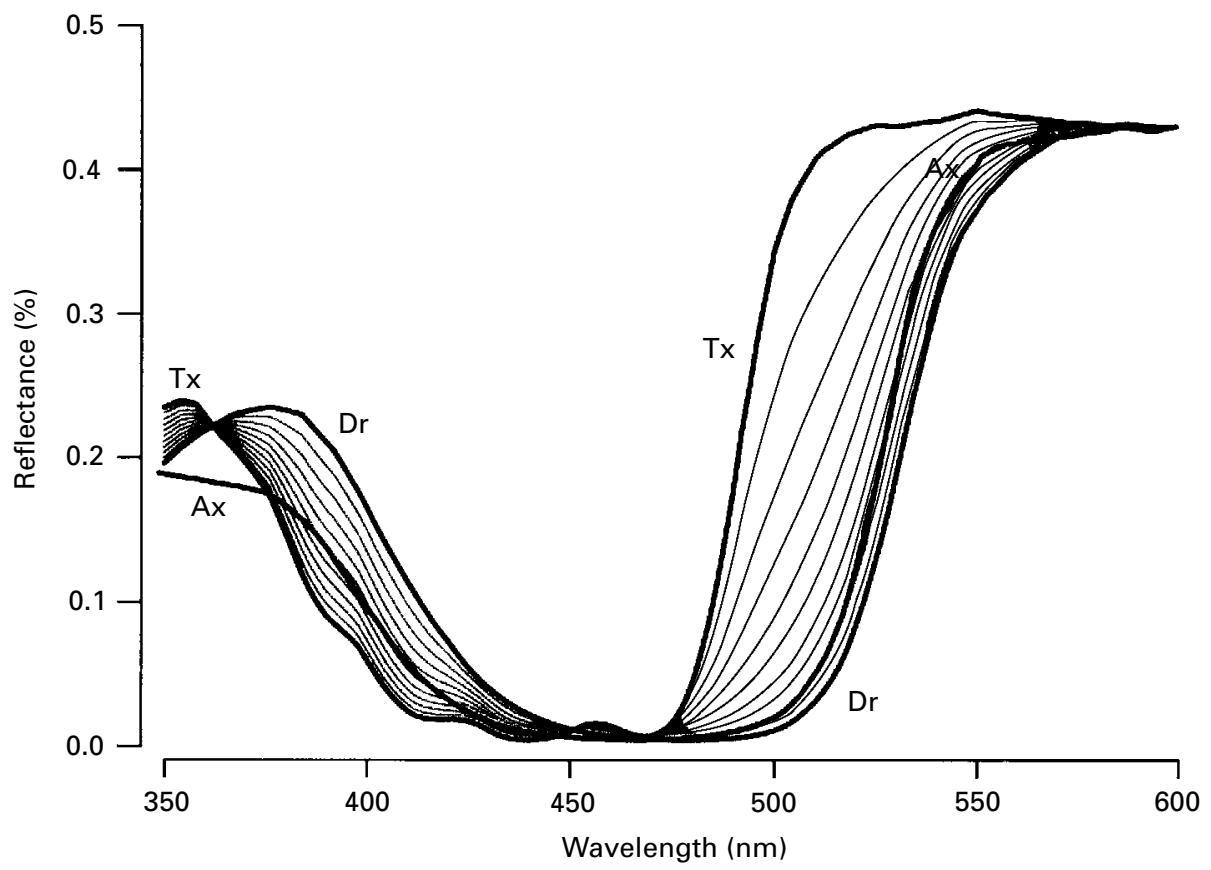

Fig. 7. Effect of xanthophore pigments on colour patch reflectance. Simulated reflectance spectra are shown for patches containing (i) only tunaxanthin (thick line labelled Tx), (ii) only astaxanthin (thick line labelled Ax), (iii) only drosopterin (thick line labelled Dr), or (iv) tunaxanthin and drosopterin in different relative amounts (unlabelled thin lines). Each of the pure pigment spectra are labelled at two points to facilitate identification. From left to right, the thin lines represent the following relative amounts of tunaxanthin (on a 0 to 1 scale): $0.9,0.8,0.7,0.6,0.5,0.4,0.3,0.2,0.1$. Note that certain tunaxanthin-drosopterin 'mixtures' closely match astaxanthin in some wavelength intervals but not in others. For the simulation results shown here, we arbitrarily set the model parameters to simulate a moderately reflective bluish-white iridophore background (reflectivity factor $r=0.5$, dilution factor $v=4$ ) with no reflective shield below the melanophores. Total xanthophore pigment concentration $\left(c_{1}+c_{2}+c_{3}\right)$ was set to 1.0.

(Fig. 8). The pigment ratio providing the closest match depended somewhat on the total amount of pigment in the xanthophore layer (see Fig. 8 legend), while the ambient light spectrum had almost no effect (results not shown). The relative cone contrast model yielded similar results, except that a 50:50 tunaxanthin-drosopterin ratio usually provided the closest match to astaxanthin (the matching ratio again depended somewhat on total pigment concentration; results not shown).

\section{(2) The amplifying effects of iridophores}

Hasson (1989) coined the term 'amplifier' for traits that have evolved because they make differences between signalers in other traits easier to detect (see Section II). Conceivably, a trait could have the same perceptual effect as an amplifer without having evolved through the amplifier process. In this section, we use amplifier terminology in reference to perceptual effects only. In Section VI, we consider whether the forms of amplification shown here are likely to be favoured by natural selection.

Consider an animal (the receiver) viewing two colour patches differing only in the carotenoid content of the xanthophore layer (model parameter $c_{i}$ ). For simplicity, we start by considering a dichromat with photoreceptor pigments absorbing maximally at wavelengths of $410 \mathrm{~nm}$ (s cone) and $543 \mathrm{~nm}$ ( $l$ cone). Because carotenoids preferentially absorb short-wave light $(<500 \mathrm{~nm})$, increases in carotenoid content of the xanthophores would reduce photon flux in the $s$ cones but have relatively little effect on the $l$ cones. Therefore, relative cone catch contrast, $D_{l s}=\left(P_{l}-P_{s}\right) /\left(P_{l}+P_{s}\right)$, and absolute cone excitation contrast, $E_{l}-E_{s}$, would both be greater for the patch with the greater carotenoid content. Iridophores underlying the xanthophores can amplify the effects of carotenoids on both of these measures of colour contrast, albeit in different ways, as illustrated by the simulations in this section.

\section{(a) Iridophore reflectivity effects}

As the reflectivity $(r)$ of an iridophore layer increases, more light will be reflected back to the receiver's eyes, after passing twice through the overlying xanthophore layer. Trivially, an unreflective iridophore layer $(r=0)$ would return no light to the receiver's eyes and thus differences in carotenoid content of the two colour patches would be imperceptible. It also seems intuitive that a highly reflective iridophore layer would make carotenoid content differences easier to detect than a poorly reflective iridophore layer (see Fig. 9). This effect cannot be demonstrated with relative cone contrasts, however, which are unaffected by changes in $r$. It is easy to see that $r$ cancels out in $D_{l s}=\left(r P_{l}-r P_{s}\right) /\left(r P_{l}+r P_{s}\right)$. 


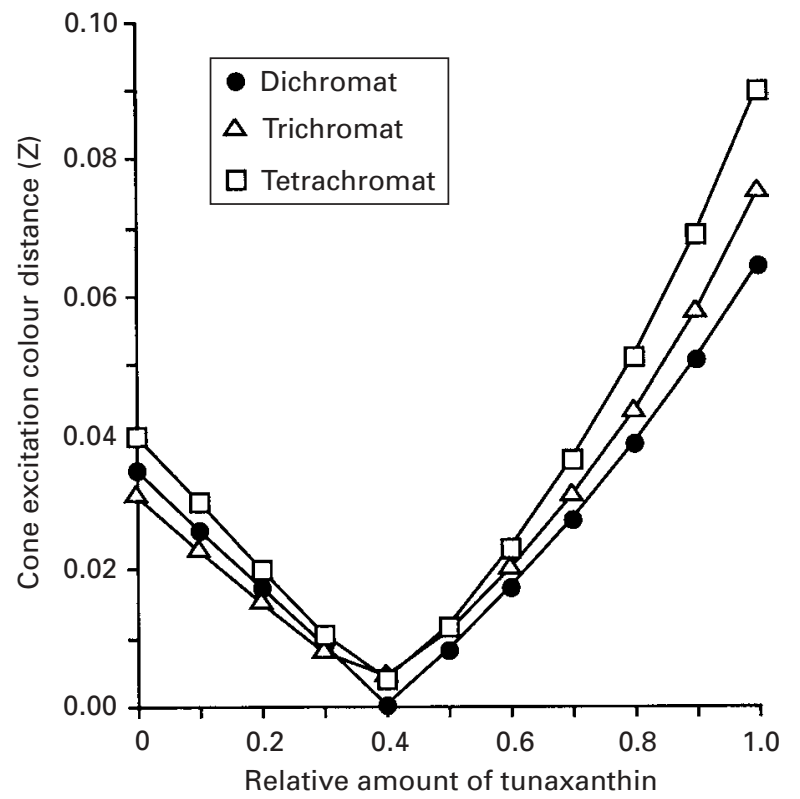

Fig. 8. The potential for spectral mimicry and fine-tuning within the xanthophore layer. This figure was generated by processing the reflectance spectra shown in Fig. 7 through the cone excitation-based vision models (equations 7-19). The vertical axis shows the distance in cone excitation colour space (Z, equation 19) between the astaxanthin-only colour patch (labelled Ax in Fig. 7) and the patches containing different relative amounts of tunaxanthin and drosopterin. Where the colour distance is minimized, the tunaxanthin-drosopterin mixture provides the closest match to astaxanthin. Here the minimum occurred at the same relative amount of tunaxanthin (0.4) for dichromatic, trichromatic and tetrachromatic receivers. Other parameter values gave slightly different results. For example, with the total xanthophore pigment concentration set to 0.1 (instead of 1.0), the minimum colour distance remained 0.4 for dichromats but was closer to 0.3 for trichromats and tetrachromats. The ambient irradiance spectrum had almost no effect on the results; here we used the open/cloudy spectrum (Fig. 4).

Whether increases in $r$ amplify or attenuate the effects of carotenoids on absolute cone excitation contrasts $\left(E_{l}-E_{s}\right)$ depends on the light spectrum to which the receiver's eyes are adapted. If the receiver's eyes are adapted to approximately the same light as the illumination light, as would often be the case in nature, then increases in $r$ always amplify the effects of carotenoids, making it easier to detect differences in carotenoid content between two patches (Fig. 10A). The situation becomes more complex if the colour patches are illuminated by light several times brighter than that to which the receiver's eyes are adapted (Fig. 10 B). If, for example, the receiver was a female perched in the shade observing two males displaying in a light gap, a difference between the males in carotenoid content might be easiest for the female to detect at intermediate levels of iridophore reflectivity. The reason for this nonlinear effect is the hyperbolic relationship between photon flux and cone excitation (Fig. 6). When the illumination light is the same as
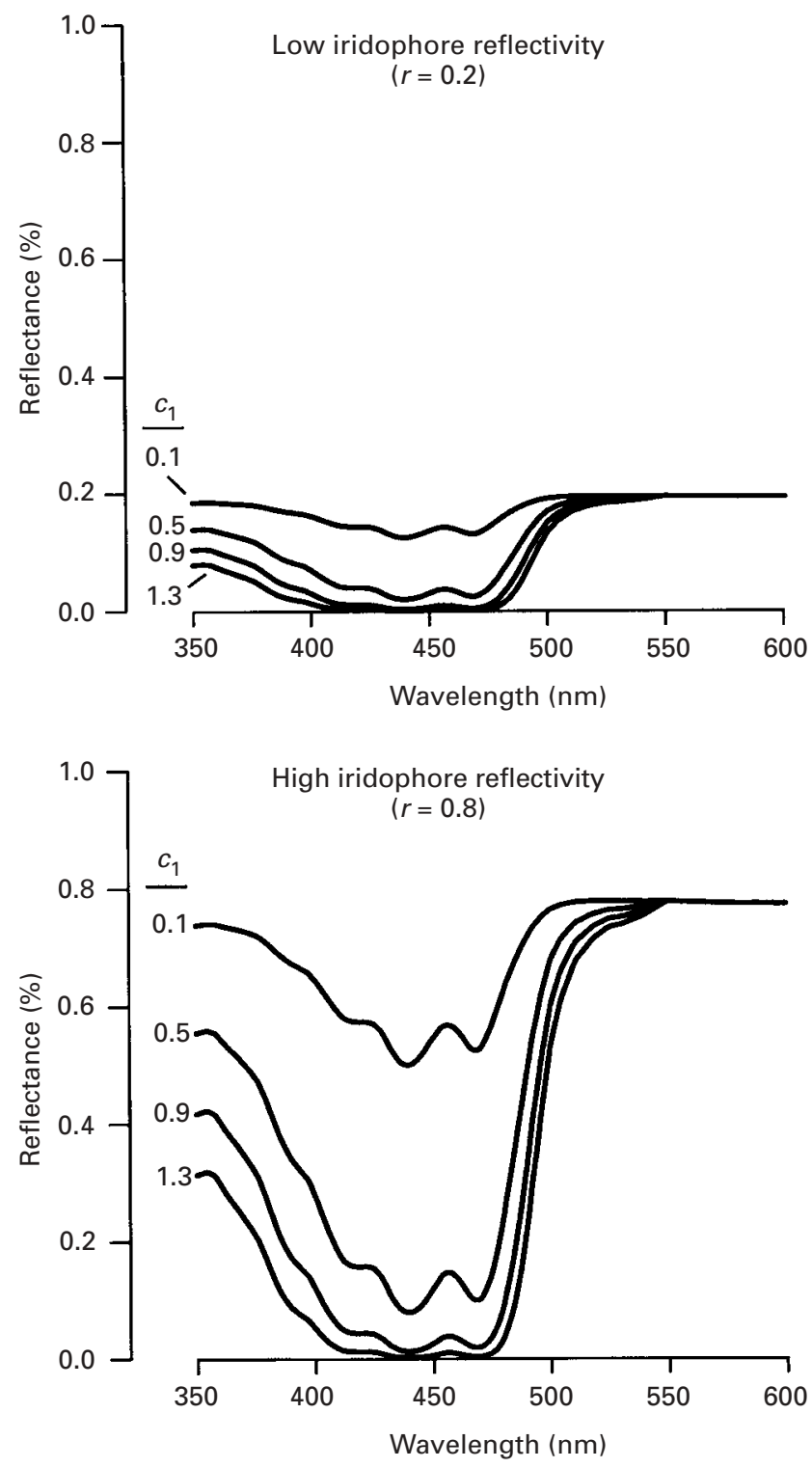

Fig. 9. The effect of xanthophore pigment (tunaxanthin) concentration $\left(c_{1}\right)$ and iridophore reflectivity $(r)$ on the reflectance spectrum of a simulated colour patch. For this simulation, we set other model parameters as follows: high iridophore dilution factor $(v=20)$, high melanin density $(d=3)$, astaxthanthin and drosopterin absent $\left(c_{2}=c_{3}=0\right)$, and reflective shield present.

the adaptation light, neither cone can reach an excitation level of greater than 0.5 and both cones remain in the steep portion of the hyperbolic intensity/response function, where changes in $r$ strongly influence cone excitation. When the illumination light is much brighter than the adaptation light, however, excitation of the $l$ cone can exceed 0.5 and enter the diminishing returns portion of the hyperbolic curve (Laughlin, 1981). Under these conditions, further increases in $r$ would reduce the difference between the $l$ and $s$ cone outputs and de-amplify the colour patch differences. To the receiver's eyes, both colour patches would converge on white. Nevertheless, regardless of the viewing conditions, 


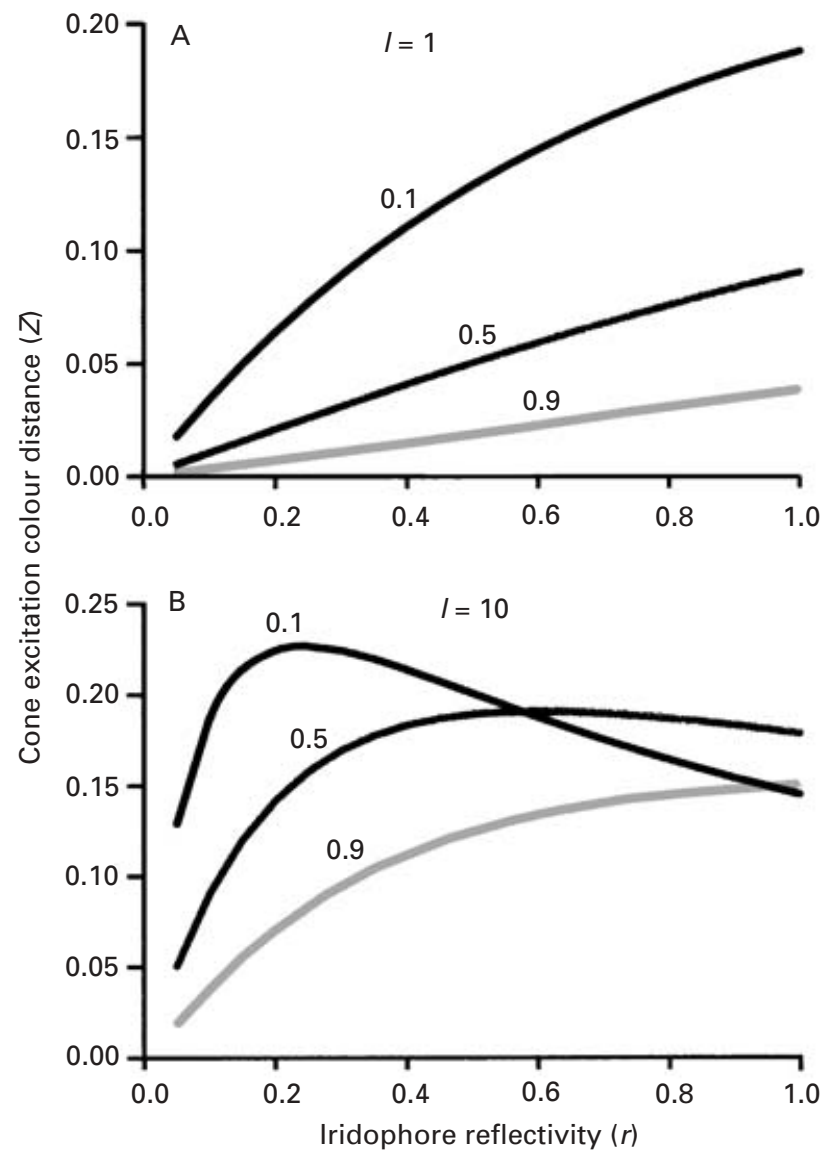

Fig. 10. The amplifying effects of iridophore reflectivity $(r)$ on variation in xanthophore pigment content $(c)$. This figure was generated by processing the reflectance spectra shown in Fig. 9 (and others for different values of $r$ ) through the cone excitationbased model. The three curves in each panel correspond to three different starting amounts of tunaxanthin $\left(c_{1}=0.1,0.5\right.$ and 0.9$)$. Colour distance $(Z)$ is the shift in cone excitation colour space (equation 19) caused by a 0.4 increase in $c_{1}$ from the indicated starting value (i.e. 0.1 to $0.5,0.5$ to 0.9 and 0.9 to 1.3). (A) Results obtained when the light illuminating the colour patch was the same as the adaptation light (illumination factor $I=1)$. In this case, increases in $r$ caused an increase in $Z$ across the full range of possible iridophore reflectivities $(0-1)$. (B) Results obtained when the illumination light was one order of magnitude greater than the adaptation light $(I=10)$. In this case, whether increases in iridophore reflectivity had an amplifying or attenuating effect on changes in xanthophore pigment content depended on the starting values of $r$ and $c_{1}$. The results shown are for dichromatic vision [short $(s)$ and long $(l)$ cones $]$, but the results for trichromats $[\mathrm{s}$, medium $(\mathrm{m})$ and $l]$ and tetrachromats [ultraviolet $(u), s, m$ and $l]$ were virtually identical.

there will always be a level of $r$ that best amplifies differences in carotenoid content of the xanthophore layer. Our simulations show that this conclusion is valid for all ambient light environments and iridophore spectra (results not shown), and that it applies equally well to dichromatic, trichromatic and tetrachromatic receivers.
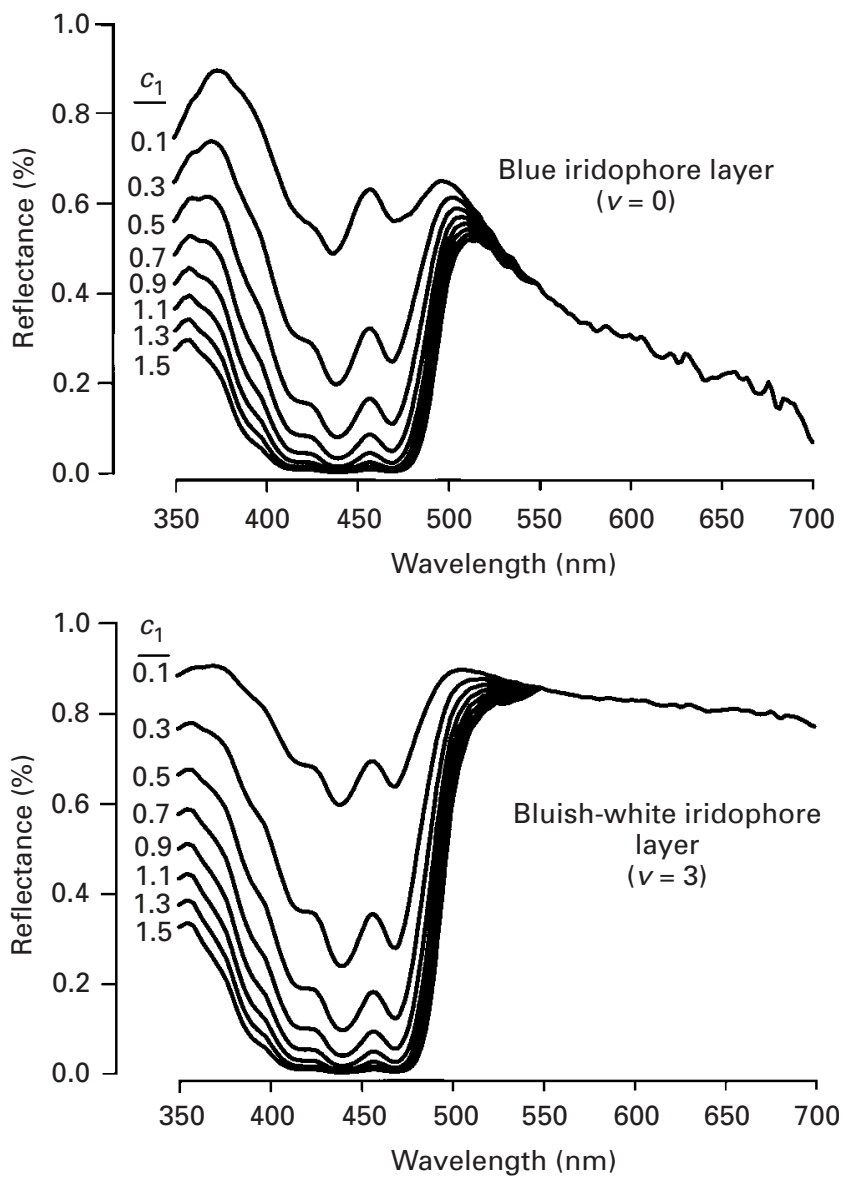

Fig. 11. The effect of xanthophore pigment (tunaxanthin) concentration $\left(c_{1}\right)$ and iridophore 'blueness' $(v)$ on the reflectance spectrum of a simulated colour patch. For this simulation, we set other model parameters as follows: maximum iridophore reflectivity $(r=1)$, high melanin density $(d=3)$, astaxanthin and drosopterin absent $\left(c_{2}=c_{3}=0\right)$, and reflective shield present.

\section{(b) Iridophore 'blueness' effects}

Increases in the ratio of short-wave to long-wave reflectance or 'blueness' of an underlying iridophore layer reduces photon catch by the $l$ cone relative to the $s$ cone, counteracting the effects of the overlying xanthophore pigments. Nevertheless, with $l$ reduced, changes in $s$ would have a larger effect on relative cone contrast $D_{l s}=\left(P_{l}-P_{s}\right) /\left(P_{l}+P_{s}\right)$. Therefore, an increase in the blueness of the iridophore spectrum (Fig. 11) would amplify the effects of xanthophore pigments on relative cone contrast (Fig. 12). The reverse is true, albeit to a trivial degree, for absolute cone excitation contrast $E_{l}-E_{s}$, because increases in carotenoid content reduce $E_{l}$ slightly and more rapidly so when $E_{l}$ is lower on the hyperbolic intensity/response curve, where changes in photon flux have a larger impact on cone excitation (results not shown).

\section{(3) The amplifying and attenuating effects of melanophores}

In species with a reflective shield of connective tissue between the skin and muscle, the melanophore layer reduces 


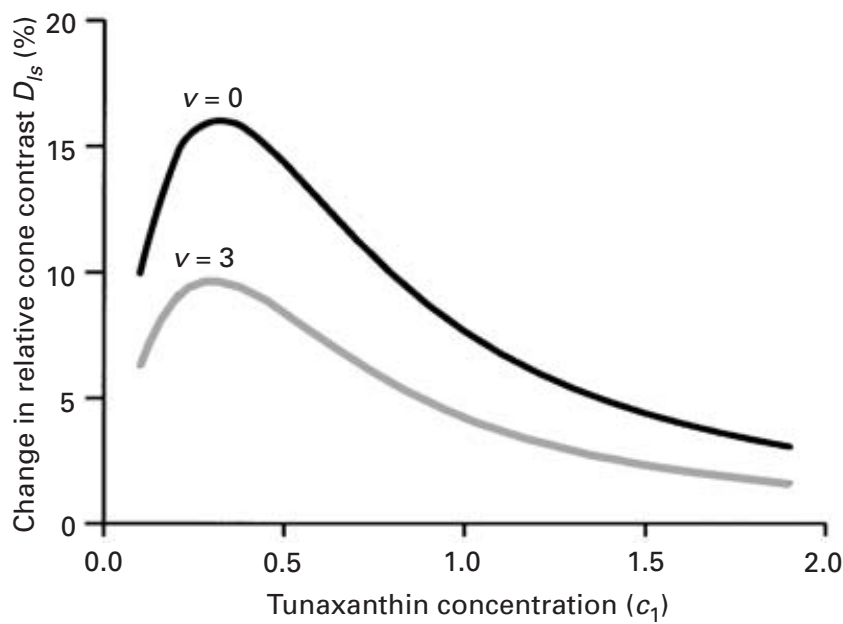

Fig. 12. The amplifying effects of iridophore 'blueness' (v) on variation in xanthophore pigment content $(c)$. This figure was generated by processing the reflectance spectra shown in Fig. 11 (and others for different values of $c_{1}$ ) through the relative cone contrast model. Each curve shows the effect of tunaxanthin content $\left(c_{1}\right)$ of the xanthophore layer on relative cone contrast $\left(D_{l s}\right)$. The vertical axis represents the percentage increase in $D_{l s}$ caused by a $50 \%$ increase in $c_{1}$ above the values shown on the horizontal axis. For example, the peak value in the upper curve indicates that $D_{l s}$ increased by $16 \%$ when $c_{1}$ increased from 0.3 to 0.45 . The vertical separation of the two curves shows that a decrease in the iridophore dilution factor $v$ amplifies the effects of $c_{1}$ on $D_{l s}$. The results shown are for open/ cloudy conditions, but the other ambient light spectra yielded similar results. The results were also similar for other values of $v$, when the $D_{\max }$ of all four guppy cones was used instead of $D_{l s}$, and when astaxanthin or drosopterin was used as the xanthophore pigment instead of tunaxanthin.

the total amount of light reflected to the receiver's eyes. In addition to reducing the overall brightness of a colour patch, melanophores modulate the relative influence of xanthophores and iridophores on the reflectance spectrum produced. Iridophores have their greatest influence, and xanthophores have their least, when the melanophores completely absorb light transmitted by the iridophores. Conversely, xanthophores have their greatest influence, and iridophores have their least, when the melanophore layer is absent and light reflected off the reflective shield passes back through the iridophores for a second round of short-wave absorption by the xanthophore pigments. Thus, melanophores have both amplifying and attenuating effects on the other chromatophore layers, as illustrated by the simulations in this section.

\section{(a) The amplifying effect of melanophores on iridophores}

Consider an animal with multiple cone types viewing two colour patches differing only in iridophore structure (parameter $v$ ). A blue iridophore layer reflects short wavelengths while allowing long wavelengths to pass through. If these long wavelengths are completely absorbed by the underlying

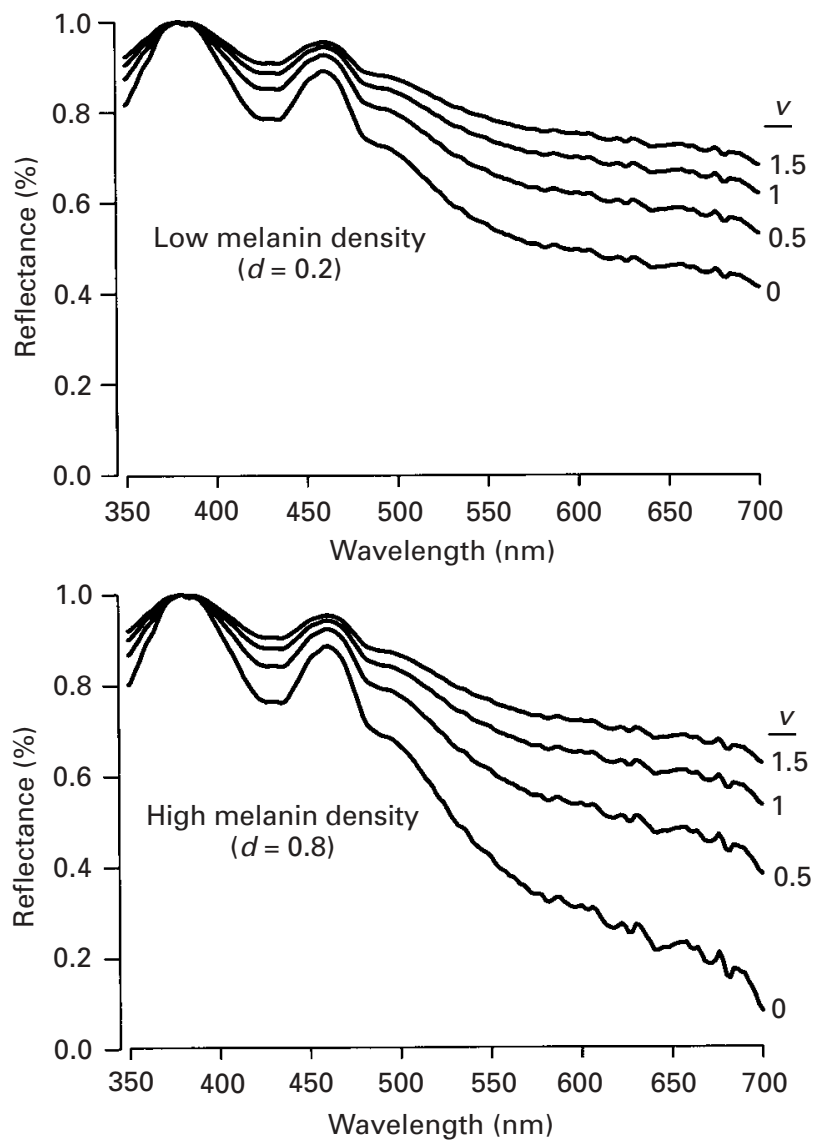

Fig. 13. The effect of melanin density $(d)$ and iridophore 'blueness' (v) on the reflectance spectrum of a simulated colour patch. For this simulation, we set other model parameters as follows: maximum iridophore reflectivity $(r=1)$, xanthophore pigments absent $\left(\right.$ all $\left.c_{i}=0\right)$, and reflective shield present.

melanophore layer (layer 3), the purity of the structural blue is preserved. However, if some light passes through the melanophores and is reflected back by the reflective shield (layer 4), the purity of the structural blue will be diluted by long-wave light (Fig. 13). Consequently, in species with a reflective shield below the melanophore layer, the melanophores amplify differences in iridophore structure. As the density of melanin (parameter $d$ in our model) increases, differences in the blueness of the iridophore layer (simulated by varying parameter $v$ ) have a larger effect on the shape of the reflectance spectrum (Fig. 13) and therefore on both absolute cone excitation contrast (Fig. 14) and relative cone contrast (results not shown). This conclusion is not sensitive to the brightness or shape of the ambient irradiance spectrum, and it holds for dichromatic, trichromatic and tetrachromatic receivers. Biologically, an increase in $d$ would be equivalent to increasing the degree of dispersion of melanosomes, increasing the density of melanosomes, or increasing the thickness of the melanophore layer. Changes in the reflectivity of the reflective shield (layer 4) have precisely the opposite effect as changes in melanin density (results not shown). 


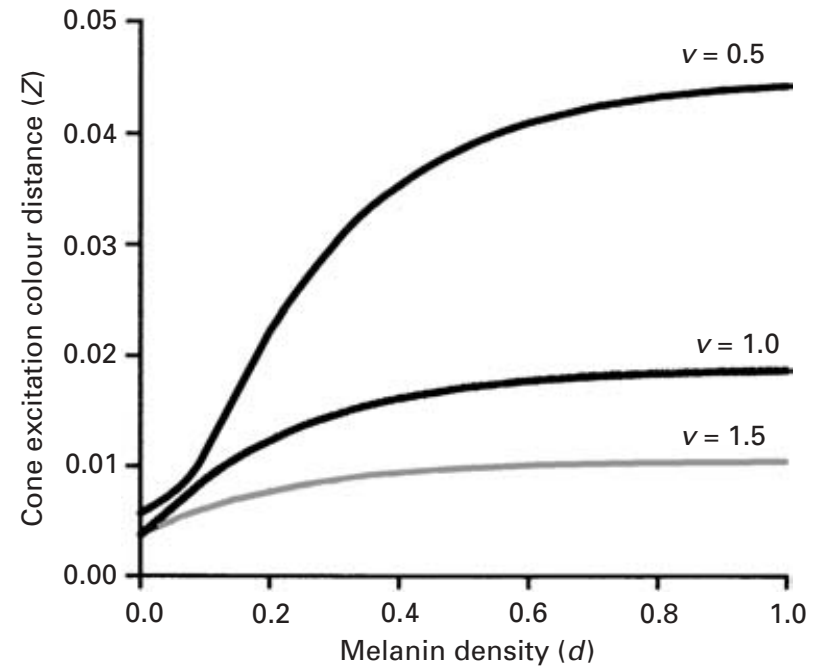

Fig. 14. The amplifying effects of melanin density $(d)$ on variation in iridophore 'blueness' (v). This figure was generated by processing the reflectance spectra shown in Fig. 13 (and others for different values of $d$ ) through the cone excitation-based model. The three curves correspond to different starting values of $v(0.5,1.0$ and 1.5). Colour distance $(Z)$ is the shift in cone excitation colour space (equation 19) caused by a 0.5 decrease in $v$ from the starting value (e.g. from 0.5 to 0 ). The results shown are for trichromatic vision ( $s, m$ and $l$ cones), but the results for dichromats $(s$ and $l$ ) and tetrachromats $(u, s, m$ and $l$ ) were quite similar. For these simulation runs, we used the open/cloudy irradiance spectrum with the illumination light equal to the adaptation light $(I=1)$, but the results were qualitatively the same with other irradiance spectra and with the illumination light tenfold brighter than the adaptation light $(I=10)$.

\section{(b) The effects of melanophores on xanthophores}

Once again, consider an animal viewing two colour patches differing only in the pigment content of the xanthophore layer (parameter $c$; see Fig. 15). If a reflective shield is present below the melanophore layer, melanophores absorb light that otherwise would be filtered twice through the xanthophores before being reflected to the receiver's eyes. Therefore, increases in melanin density $(d)$ tend to attenuate (de-amplify) the effects of xanthophore pigments on absolute cone excitation contrasts. This is always the case when the viewer's eyes are adapted to the same intensity of light as illuminates the colour patch (Fig. 16A), but when the colour patch is more brightly illuminated than the viewer's eyes, changes in melanin density can have the opposite effect (Fig. 16B). The cause of this transient reversal under bright lighting lies in the hyperbolic relationship between photon flux and cone excitation (Fig. 6), for reasons analogous to those described in Section V.2a. These results were not influenced qualitatively by the shape of the iridophore reflectance spectrum (parameter $v$ ).

The relative cone contrast method leads to somewhat opposite conclusions. If the iridophore reflectance spectrum is spectrally flat (high $v$ ), then changes in melanin density have no effect on the difference between the two colour patches in relative cone contrast (Fig. 16G). However, if the iridophores preferentially reflect short-wave light (low v), then increases in melanin density $(d)$ amplify the carotenoid content differences (Fig. 16D). The reason for this is the same as described above under iridophore 'blueness' effects (Section V.2b). Melanophores differentially reduce long-wave reflectance relative to short-wave reflectance, thereby amplifying the short-wave-absorbing effects of carotenoids.

\section{(4) Summary of simulation results}

The basic properties of the dermal chromatophore unit model are fairly well known (reviewed in Section III). The purpose of our simulations was to explore the potential for interactions within and between the chromatophore layers in terms of their effects on colour perception. The first simulation illustrated the potential for spectral fine-tuning and mimicry within the xanthophore layer. The orange-red carotenoid astaxanthin can be matched almost perfectly by combining a red pteridine (drosopterin) with a yellow carotenoid (tunaxanthin), holding total pigment concentration constant (Fig. 8). The possible evolutionary implications of this result are discussed below (Section VI.1).

Other simulations examined whether changes in the properties of inner chromatophore layers amplify or attenuate the visual effects of changes in outer chromatophore layers. The results depended somewhat on assumptions about visual processing and viewing conditions. Intuitively, an increase in iridophore reflectivity should make differences between colour patches in xanthophore pigment concentration easier to detect (see Fig. 9). This would not be the case, however, if photon capture was converted into relative cone contrasts before high-level visual processing in the brain, as is often assumed. Chittka's (1992) absolute cone excitation method yielded the expected result (amplification) under 'normal' viewing conditions (Fig. 10A). This method also revealed that increases in iridophore reflectivity can have the opposite effect (attenuation) if the colour patches to be compared are illuminated by light substantially brighter that that to which the receiver's eyes are adapted (Fig. 10B). Another way iridophores can amplify xanthophores is through changes in the shape or 'blueness' of the iridophore reflectance spectrum. An increase in iridophore 'blueness' reduces photon capture by long-wave cones relative to short-wave cones and therefore amplifies the effects of xanthophore pigments on relative cone contrasts (but not absolute cone excitation contrasts) (Fig. 12).

The final set of simulations examined how changes in melanophore absorption modify perception of changes in iridophore 'blueness' and xanthophore pigment concentration. Regardless of lighting conditions and visual processing mechanisms, melanophores amplify the spectral effects of iridophores (Fig. 14). The relationship between melanophores and xanthophores is more complicated. Under 'normal' viewing conditions, melanophores attenuate the effects of xanthophore pigments on absolute cone excitation contrasts (Fig. 16A), but under bright illumination the reverse effect (amplification) is possible (Fig. 16 B). With the relative cone contrast model, melanophores 

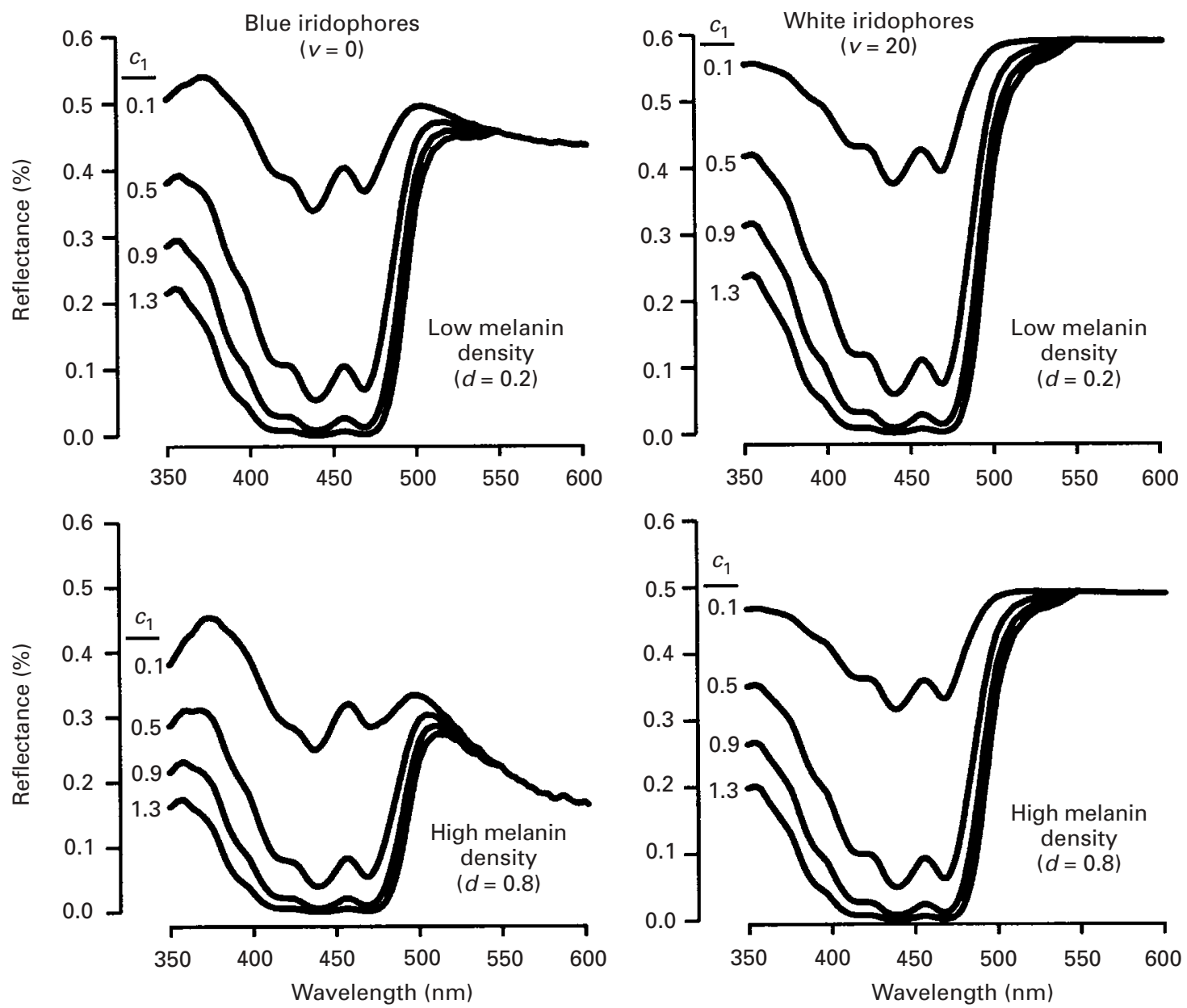

Fig. 15. The effects of xanthophore pigment (tunaxanthin) concentration $\left(c_{1}\right)$, melanin density $(d)$, and iridophore 'blueness' $(v)$ on the reflectance spectrum of a simulated colour patch. For this simulation, we set other model parameters as follows: moderate iridophore reflectivity $(r=0.5)$, astaxanthin and drosopterin absent $\left(c_{2}=c_{3}=0\right)$, and reflective shield present.

can amplify the effects of xanthophores, but only if the iridophore layer preferentially reflects short-wave light (Fig. 16C, D).

In general, the results of our simulations were affected only slightly by the ambient light spectrum and the number of cones included in the calculations. Where the two methods for calculating colour contrasts yielded conflicting results, we view the absolute cone excitation results as more relevant for signal evolution than the relative cone contrast results, as explained below (Section VI.2c).

\section{INDIVIDUAL GOLOUR PATGHES AS MULTICOMPONENT TRAITS}

In this section we synthesize the material of the preceding sections, with the primary goal of showing how multiple trait evolution theory can be applied to the evolution of multicomponent colour patches.

\section{(1) Xanthophores}

(a) Carotenoids versus pteridines

Because of their exogenous (dietary) origin, carotenoids have long been viewed as honest signals of foraging ability that females could use to choose high-quality mates (Endler, 1980). Carotenoids may also reveal an individual's history of disease, current state of health, or potential to respond to future infections, through physiological effects of parasites on carotenoid metabolism, or utilization and oxidation of carotenoids by the immune system (Kodric-Brown \& Brown, 1984; Putnam, 1992; Hudon, 1994; Lozano, 1994; Thompson et al., 1997; Olson \& Owens, 1998; Wedekind et al., 1998; Camplani, Saino \& Møller, 1999; von Schantz et al., 1999; Møller et al., 2000). The pro-vitamin A activity of some carotenoids is well known (Goodwin, 1986). Carotenoids have also been implicated as antioxidants, immune response enhancers, and cancer inhibitors (Bendich, 1989; Burton, 1989; Connett et al., 1989; Krinsky, 1989; Møller et al., 2000; Blount et al., 2003; Faivre et al., 2003; Grether 

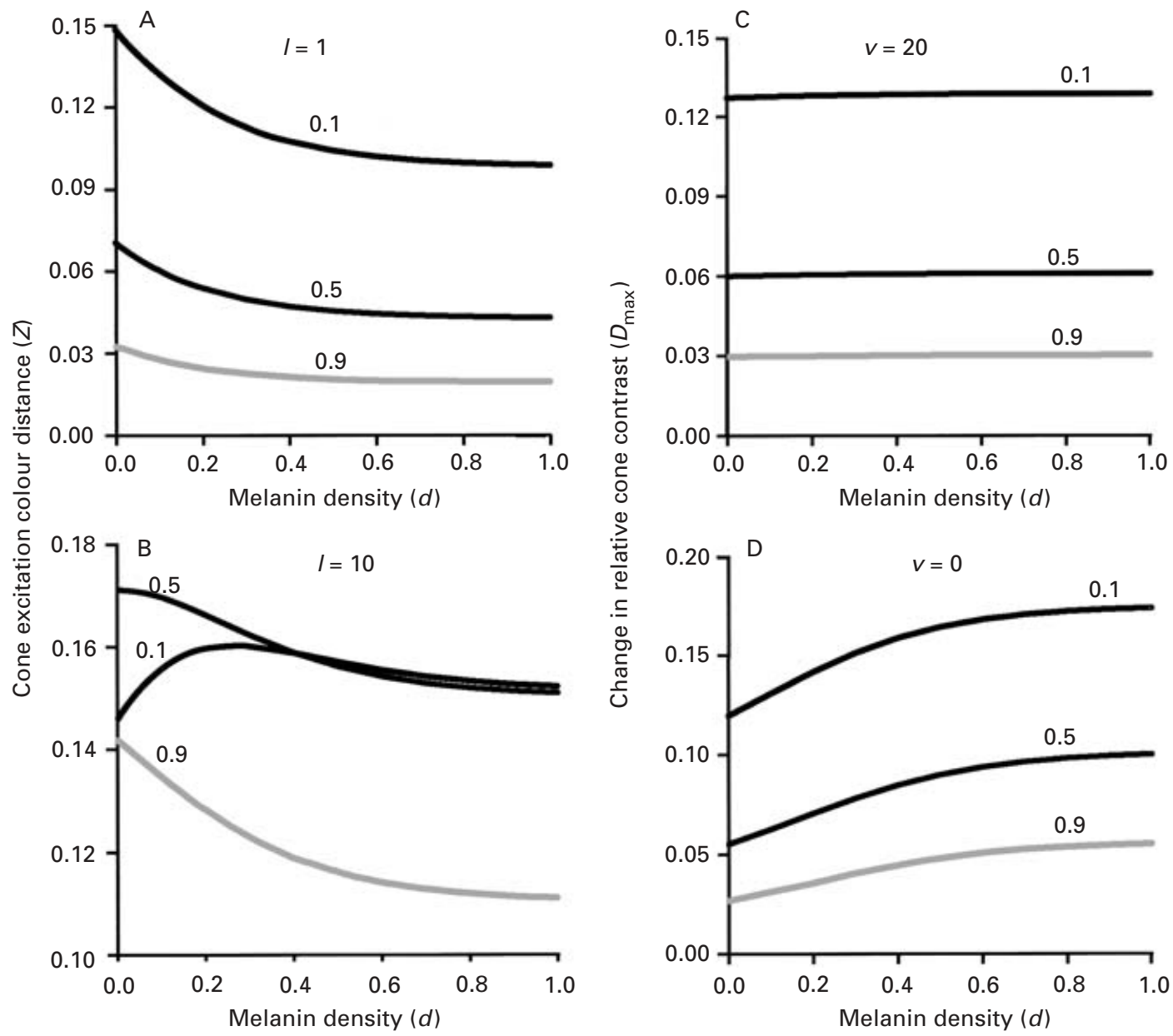

Fig. 16. The effects of melanophores on perceived differences in xanthophore pigment content. This figure was generated by processing the reflectance spectra shown in Fig. 15 (and others for different values of $d$ ) through the visual system models. Each line shows the change in 'colour' caused by a 0.4 unit increase in tunaxanthin content $\left(c_{1}\right)$ above the indicated value $(0.1,0.5$ or 0.9$)$ as melanin density $(d)$ varies from 0 to 1 . A and $\mathrm{B}$ show the results for absolute cone excitation contrasts; $\mathrm{C}$ and $\mathrm{D}$ show the results for relative cone contrasts. Comparison of A and B shows that the effect of melanin density on the change in absolute cone excitation contrasts depends on the intensity of light illuminating the colour patch. The illumination light is the same as the adaptation light in A (illumination factor $I=1$ ) and one order of magnitude brighter than the adaptation light in $\mathrm{B}(I=10)$. The shape of the iridophore spectrum had no qualitative effect on these results $(v$ was set to 0 for the simulations shown here, but the results were qualitatively the same with $v=20)$. By contrast, the shape of the iridophore spectrum did influence the effect of melanin density on relative cone contrasts. G shows the results for a spectrally flat iridophore spectrum $(v=20)$, while D shows the results for a 'blue' iridophore spectrum $(v=0)$. Note that relative cone contrasts are insensitive to illumination intensity. The results shown here are for tetrachromatic vision; the results for dichromats and trichromats were similar. Iridophore reflectivity $(r)$ was set to 0.5 for these simulations (the same patterns hold for $0<r<1.0$ ). The open/cloudy irradiance spectrum was used in these simulations; other ambient light spectra yielded similar results.

et al., in press; but see Hill, 1999). Pteridines, by contrast, are endogenously produced from purines, which in turn are synthesized de novo from carbohydrates and proteins (Hurst, 1980), and have no known links with health. It is possible, however, that pteridine production entails significant metabolic costs.

The presence of spectrally similar pigments with different biochemical origins within a single colour patch may provide opportunities for spectral mimicry, e.g. of carotenoids by pteridines, and thus of deception of females by low-quality males. Under this scenario, pteridines would be unreliable signals and carotenoids would be handicaps (Table 1). In theory, however, either class of pigments could be a cheap substitute for the other, depending on the diet and physiology of the animal. Alternatively, exogenous carotenoids and endogenous pteridines could represent parallel indicator systems if they reflect different aspects of mate quality (e.g. foraging ability and developmental stability), or redundant indicator systems if they reflect similar aspects of mate quality (e.g. overall health). The 
latter two hypotheses are analogous to the multiple message and backup/redundant signal hypotheses, except that females would not have to evolve a separate preference for each ornament (see also Brooks \& Couldridge, 1999). A single preference for chroma (colour saturation) or hue (spectral shape) could exploit carotenoid- and pteridinebased indicators simultaneously. This is a situation in which the joint costs of mate choice, which can constrain the evolution of multicomponent displays (Iwasa \& Pomiankowski, 1994), should be quite low.

The existence of pigments with different absorptive properties in the xanthophore layer may allow for greater spectral fine-tuning than could be accomplished with one pigment alone (see Figs 7 and 8). Spectral fine-tuning could be an adaptation for matching the background (crypsis), for contrasting maximally with the background (conspicuousness), or for matching a sensory bias in the receiver's visual system. Spectral fine-tuning could also potentially evolve through the indicator process if the metabolic capacity to coordinate the production of two different pigments is an honest signal of quality ('pigment symmetry').

These ideas have begun to be tested in guppies (Poecilia reticulata). The xanthophores of male guppies contain red pteridines (drosopterins) and yellow carotenoids (primarily tunaxanthins), which together produce the orange spots that have captured the interest of many biologists. Female guppies prefer males with higher orange chroma (KodricBrown, 1989, 1993; Houde \& Torio, 1992; Grether, 2000) and greater orange spot area (Houde \& Endler, 1990; Endler \& Houde, 1995; Jirotkul, 1999), but the carotenoid content of the orange spots is constrained by carotenoid availability, which varies among streams (Grether, Hudon \& Millie, 1999). The spectral mimicry hypothesis predicts that males should produce more drosopterins in streams where carotenoid availability is lower, but precisely the reverse pattern was observed in a comparison of guppies from six streams differing in carotenoid availability (Grether et al., 2001 a). This result would be consistent with the parallel indicator (multiple message) hypothesis if drosopterin production is metabolically costly and sensitive to food intake. Food availability and carotenoid availability are closely linked for guppies, because unicellular algae are the primary food source as well as the primary carotenoid source (Grether et al., 1999, 2001 b). Whether drosopterin production is condition-dependent remains to be determined, but the available evidence suggests that the stream differences in drosopterin production are largely genetic (see Grether et al., 2001 a). Another possible explanation for the positive correlation between carotenoid and drosopterin content of the orange spots across streams is that female guppies prefer a particular hue of orange. Under this hypothesis, males would be selected to match the deposition of yellow carotenoids with a complementary amount of red drosopterins. A hue preference could be a female counter-adaptation to males 'cheating' with drosopterins in a carotenoid-indicator system, or it could reflect a preexisting sensory bias (Grether et al., 2001a).

Under the pre-existing hue preference scenario, guppies might use tunaxanthins and drosopterins instead of a single orange carotenoid because the dual pigment system is less costly in a carotenoid-limited environment. No poeciliids use orange 4-keto-carotenoids, but the metabolic capacity to convert dietary carotenoids into 4-keto-carotenoids does exist in this group (certain hybrids of Xiphophorus produce astaxanthin; Rempeters, Henze \& Anders, 1981). Perhaps the dual pigment system evolved to replace the use of a single orange carotenoid, and the capacity to produce orange carotenoids was secondarily lost or suppressed in this group (Hudon et al., in press).

\section{(b) Multiple carotenoids}

Although incapable of producing carotenoid pigments de novo, animals can convert ingested carotenoids into pigments with very different spectral or absorptive properties before displaying them in the integument (Goodwin, 1984; Brush, 1990). Why do particular species display particular carotenoids or combinations of carotenoids? To what extent does the distribution of carotenoid use reflect constraints imposed by diet or phylogeny? How much of the variation in carotenoid use can be explained in terms of indicator processes and sensory drive? Although some of the proximate causes of species differences in carotenoid colouration are understood at a basic level (see Inouye et al., 2001; McGraw et al., 2001; Stradi, Pini \& Celentano, 2001), the evolutionary questions remain open (Hudon et al., in press).

Some carotenoid-based colours may be more costly to display than others, depending on the dietary availability of different carotenoid pigments, and the number of biochemical steps required to convert ingested carotenoids into integumentary pigments. Red keto-carotenoids tend to be less abundant than yellow carotenes and xanthophylls in the diet of most vertebrates (Hill, 1996). Costs of metabolic conversion may make red carotenoids more costly, and thus more reliable as indicators of phenotypic quality, than yellow carotenoids (Hudon, 1991; Hill, 1996). This hypothesis appears to be supported by Hill's (1996) comparative study of cardueline finches (the degree of sexual dichromatism and the redness of male plumage correlate positively across species in this clade), and also by a food limitation study (controlling for carotenoid intake, better fed house finches Carpodacus mexicanus were redder; Hill, 2000).

There is some evidence that dietary carotenoids boost the immune systems of vertebrates with carotenoid colouration (Møller et al., 2000; Blount et al., 2003; Faivre et al., 2003; Grether et al., in press; but see Hill, 1999; Lozano, 2001). Different carotenoids may provide different information to receivers (multiple messages) if, for example, they are used by the immune system to combat different types of infections. Wedekind et al. (1998) offered this as a possible explanation for the presence of multiple pigments in the ventral colouration of three-spined sticklebacks (Gasterosteus aculeatus). As in guppies, the nuptial colouration of male sticklebacks is produced by a combination of red and yellow pigments, except that, in sticklebacks, both types of pigments are carotenoids (astaxanthin and tunaxanthin and/or lutein). Whether these two types of pigments affect the immune system of sticklebacks differently remains to be determined. 


\section{(2) Iridophores}

\section{(a) Detectability}

Iridophores play the vital role of reflecting light into the receiver's eyes. Generally speaking, the detectability of a stimulus increases with its brightness (perceived intensity) relative to the background. For example, Persons et al. (1999) and Fleishman \& Persons (2001) showed, in anoles, that an increase in brightness of a moving, dewlap-like stimulus relative to the background increased the likelihood of detection of the stimulus. In this case, the detection response was a reflexive action independent of subsequent responses based on stimulus content. Detectability has also been shown to increase with stimulus brightness in honeybees Apis mellifera (De Ibarra et al., 2000). Changes in iridophore reflectivity may be one of the primary mechanisms through which a selective balance is reached between the benefits of conspicuousness in intraspecific communication, on the one hand, and the benefits of crypsis in a predatorprey context, on the other.

\section{(b) Indicator value}

In addition to modulating brightness, iridophores also have the potential to function as quality indicators and amplifiers. The wavelength composition and proportion of incident light reflected by an iridophore layer depends critically on the precise thickness and spacing of reflecting platelets at the nanometer scale (Huxley, 1968; Denton \& Land, 1971; Herring, 1994; also see Section III). As has been argued for the structural-colour-producing mechanisms in bird feathers (Fitzpatrick, 1998b), the ultrastructural perfection and uniformity of iridophores may be affected by, and therefore function as an indicator of, developmental stability. This hypothesis could be tested by examining the effects of destabilizing factors (e.g. environmental stressors, parasites, inbreeding and age) on iridophore reflectance, and perhaps also by examining correlations between iridophore reflectance and other putative correlates of developmental stability, such as fluctuating asymmetry (Parsons, 1990; Møller \& Thornhill, 1998; Thornhill, Møller \& Gangestad, 1999; but see Palmer, 1999, 2000; Lens et al., 2002).

\section{(c) Xanthophore amplification}

Our simulations show that changes in the properties of the iridophore layer can make differences between colour patches in xanthophore pigment content easier or harder for receivers to detect (see Section V.2). This is not surprising, given that, in the absence of a reflective layer, differences between signalers in xanthophore pigment content would be impossible to detect. Whether the properties of iridophores could evolve through the amplifier process, however, is a separate question. Hasson (1989) coined the terms 'amplifier' and 'attenuator' for traits that originally evolved because they made differences between signalers in indicator traits easier or harder to detect, respectively (Table 1). For example, the barred markings on bird feathers have been proposed to be amplifiers of feather growth irregularity and thus of developmental instability (Hasson, 1991).
Conceivably, however, a trait could have the perceptual effect of making differences between signalers in other traits easier to detect without having evolved for this purpose. One example of this may be the amplification of the effects of xanthophore pigments on relative cone contrasts caused by increases in iridophore 'blueness' (Figs 11 and 12). Let's assume that females prefer males whose colour patches contain higher concentrations of carotenoids, as is the case, for example, in guppies (Kodric-Brown, 1989; Grether, 2000), and that females use relative cone contrasts (e.g. $D_{l s}$ ) for comparing males. The simulations reveal that an increase in the 'blueness' (reduced $v$ ) of the iridophore layer would make it easier for females to distinguish between males with different carotenoid concentrations. To evaluate whether this sort of amplification could evolve, however, we need to consider the starting conditions. All else being equal, a high carotenoid patch would produce a higher value of $D_{l s}$ than a low carotenoid patch. A mutant male with a blue-shifted iridophore spectrum below the xanthophore layer would therefore be at an obvious disadvantage (lower $D_{l s}$ ). In this case, female choice would favour a mutation in the opposite direction, towards reduced amplification (higher $v$ ). This line of reasoning leads to the prediction that iridophore layers with overlying xanthophores should be spectrally flat (or red-shifted), in comparison to iridophores without overlying xanthophores. To our knowledge, no data are currently available to test this prediction, but it could be tested by measuring the reflectance properties of individual iridophores (Fujii et al., 1991; Morrison, 1995; Morrison et al., 1995).

Although spectral amplification of xanthophores by iridophores would not be evolutionarily stable, brightness amplification would be evolutionarily stable if females compared males using absolute cone contrasts (e.g. $E_{l}-E_{s}$ ). All else being equal, a high carotenoid patch would produce a higher value of $E_{l}-E_{s}$ than a low carotenoid patch. A mutation for increased reflectivity $(r)$ of the underlying iridophore layer would increase $E_{l}-E_{s}$ and therefore increase the mutant male's attractiveness relative to males with the same carotenoid concentrations but lower reflectivity values. Once the mutation spread through the population, and all males possessed the same high value of $r$, females would find it easier to distinguish between males with different carotenoid concentrations (Fig. 10A).

Nevertheless, the sort of amplification that occurs between layers within a colour patch is qualitatively different from that described by Hasson (1989, 1990, 1991). By his definition, an amplifier 'always decreases the mating success of the less viable males' (Hasson, 1989). In the scenario described above, however, males of all viabilities (carotenoid concentrations) would benefit from a mutation for increased iridophore brightness, and no male would benefit from a mutation in the opposite direction. Once the mutation for increased brightness spread through the population, low carotenoid males would suffer because females could more easily identify the high carotenoid males, but there would still be no advantage to low carotenoid males of reducing the reflectivity of their iridophores. By contrast, with Hasson's amplifiers, modifier genes that cause the amplifier to be suppressed in low-viability males would be favoured. 
Once these modifier genes spread through the population, females could use the amplifier trait itself to discriminate against low-viability males. Thus, at equilibrium, the amplifier would also be an indicator of viability, not a pure amplifier (Hasson, 1990, 1991). The sort of amplification that occurs between layers of a colour patch may therefore be the purest form of amplification yet described.

The level of iridophore reflectivity that maximizes amplification of xanthophore differences is not necessarily the theoretical maximum (i.e. $r=1.0$ ). When the receiver's eyes are adapted to a dimmer spectrum than the one illuminating the colour patch, amplification is greatest at intermediate levels of iridophore reflectivity (Fig. 10B). One prediction that follows from this observation is that species that display in light gaps should have less reflective iridophores underlying their xanthophores than do species that display under most other lighting conditions (e.g. open, cloudy, shady). Thus, depending on signalling conditions, the amplifier process can lead to either directional or stabilizing sexual selection on iridophore reflectivity. The same predictions may be applied to the reflective structures underlying carotenoid pigmentation in bird feathers.

Do animals actually use absolute cone excitation contrasts for making colour discriminations? Traditionally, absolute cone inputs are factored out of colour discrimination measures, and total cone input is treated as a separate, achromatic brightness channel (see Rodieck, 1973; Endler, 1991 b). Chittka (1992) criticized this practice and reviewed evidence suggesting that the ability of animals to make colour discriminations is affected by light intensity. For example, it is well known that, for human receivers, all colours converge on black as illumination intensity decreases and on white as illumination intensity increases. Chittka's method of calculating colour contrasts based on absolute cone excitations may not be perfect (Chittka, 1999; Vorobyev et al., 1999), but to our knowledge, the premise that brightness affects colour discrimination has not been challenged.

\section{(d) Xanthophore mimicry and complementarity}

Contrary to popular belief, iridophores do not always produce blue or white (silvery) reflectance spectra. Depending on iridophore ultrastructure, almost any colour may be produced, including yellow, orange and red (Fujii, 1993). The notion that structural colours in animals are limited to blues and blue-greens may be due, in part, to the outdated assumption that such colours are produced by Tyndall scattering (e.g. see Bagnara \& Hadley, 1973). Through thinlayer interference, iridophores have the potential to complement the spectral effects of xanthophore pigments. Recall that in the orange morph of Sceloporus undulatus erythrocheilus, xanthophore pigments are red and iridophores reflect an orange spectrum, while in the yellow morph, xanthophore pigments are yellow and iridophores reflect a yellow spectrum (Morrison et al., 1995). From a signal evolution standpoint, this outcome makes perfect sense. Morphs with mismatched xanthophores and iridophores would probably be at a selective disadvantage. This example is also intriguing from a developmental perspective, because it implies ontogenetic linkage between xanthophore pigments and iridophore ultrastructure. The matching xanthophores and iridophores found in the different morphs may simply represent different ways of being optimally conspicuous. However, if the xanthophore pigments are quality indicators in this species, the complementary iridophore spectrum may have evolved through spectral mimicry. Under this evolutionary scenario, the iridophores could also be viewed as attenuators, because a complementary reflectance spectrum would make differences in xanthophore pigment content difficult to detect. Both high and low quality males would benefit from a mutation for a complementary iridophore spectrum, but only low quality males would benefit after the mutation spread to fixation. We predict that as more studies of colour production mechanisms at the cellular level are conducted, complementary xanthophores and iridophores will turn out to be common if not the norm.

\section{(3) Melanophores}

Melanophores are widely recognized for their role in adaptive colour change and crypsis. These melanincontaining cells are responsible, for example, for disruptive banding patterns and rapid darkening and lightening of the integument (Bagnara \& Hadley, 1973; Fujii, 2000). Less appreciated is the role of melanophores in colour production, per se. In species with a basal reflective shield of connective tissue, melanophores determine how much of the light that escapes through the xanthophore and iridophore layers is reflected back for a second round of absorption by xanthophore pigments (see Section III). As such, melanophores modulate the relative influence of the iridophores and xanthophores, particularly when these cells have contrasting, as opposed to complementary, effects on the final reflectance spectrum. The influence of iridophores is greatest when melanophores absorb all light that escapes through the iridophores. This is why the extra layer of melanin in the structural blue dewlap of Anolis conspersus makes the colour 'deeper' (Macedonia, 2001). Conversely, the influence of xanthophores is greatest when the melanophore layer is absent and the reflective shield is highly reflective, as illustrated by $A$. grahami, the putative ancestor of A. conspersus with the orange dewlap (Macedonia, 2001).

In a sexual signalling context, melanophores could function as amplifiers of iridophore ultrastructure, which, as noted above, may be an indicator of developmental stability. Our simulations show that increases in melanophore absorption would make differences between two colour patches in the spectrum reflected by the iridophores easier for receivers to detect, regardless of ambient lighting conditions (Figs 13 and 14). This form of amplification should be evolutionarily stable. If females preferred males with more chromatic structural colours, a mutant male with a greater density of melanin below the iridophore layer would have an immediate advantage (higher cone contrast). After such a mutation spread through the population, the attractiveness differential between high and low quality males would be wider than before, but modifier genes suppressing the mutant gene in low quality males would not be favoured. Thus, melanophores could function as pure amplifiers of iridophore ultrastructure. 
The relationship between melanophores and xanthophores is complicated (Figs 15 and 16), but the bottom line is probably that melanophores would rarely be evolutionarily stable as amplifiers (or attenuators) of xanthophore differences. Increases in melanophore absorption might make differences between two xanthophore patches easier to detect by a receiver using relative cone contrasts (see Fig. 16D), but neither low nor high quality signalers would benefit from a mutation in this direction. This is because an increase in melanophore absorption would reduce the relative cone contrast (i.e. chroma) of a xanthophore patch. Normally, increases in melanophore absorption would make differences between two xanthophore patches harder to detect by a receiver using absolute cone contrasts. The exception is when the colour patches are illuminated by light much brighter than that to which the receiver's eyes are adapted (Fig. 16B). In this situation, an intermediate (non-zero) level of melanophore absorption might be optimal, both for maximizing the cone contrast caused by a given concentration of xanthophore pigments and also for maximizing the perception of xanthophore differences.

Based on these considerations, we would expect to see well-developed melanophore layers in species that use iridophore structure as a criterion in mate choice. Conversely, melanophores should be reduced (or absent) when mate choice is based primarily on xanthophore pigment content, except, perhaps, for species displaying in light gaps (see Fig. 16B). The same predictions could be applied, of course, to colour patches used as indicators in other contexts, e.g. territory competition (see Grether, 1996).

Some additional predictions are possible for species with motile melanophores. Light absorption by melanophores is greatest when the melanosomes within these cells are dispersed and least when the melanosomes are aggregated. By keeping melanosomes aggregated at some times and dispersed at other times, signalers could potentially strike a more optimal balance between the costs and benefits of conspicuous colouration. When the benefits (e.g. sexual attractiveness) depend on xanthophore pigment content, melanosomes should be aggregated during relevant interactions with conspecifics (e.g. courtship) and dispersed at other times. Conversely, when the benefits depend on iridophore ultrastructure, melanosomes should be dispersed during interactions with conspecifics and aggregated at other times. In species with both xanthophore- and iridophore-dominated patches within their overall colour pattern (e.g. guppies), the melanosomes should be aggregated under the iridophore patches while they are dispersed under the xanthophore patches, and vice versa (assuming the different patches have similar functions, such as attracting mates).

\section{GONGLUSIONS}

(1) Colour patches are composite traits, the cellular and subcellular components of which may evolve independently. The theory of multiple trait evolution, which was developed to explain multiple ornaments, can also be applied to the multiple components of single colour patches. Colour patches can be viewed as ornaments nested within ornaments.

(2) The presence of pigments with different biochemical origins in the xanthophore layer creates opportunities for spectral fine-tuning and mimicry. Signalers are expected to mimic favoured colours using the least costly alternatives (e.g. substituting endogenously produced pteridines for carotenoids if the latter are environmentally limiting). Receivers may in turn evolve perceptual mechanisms (e.g. hue preferences) to circumvent this type of cheating. The end game may be a stalemate of sorts, where an evolutionarily stable combination of pigments is used.

(3) Inner chromatophore layers can have the perceptual effect of amplifying (or attenuating) variation in outer chromatophore layers. Some of these effects depend on viewing conditions (ambient light) and visual processing mechanisms in the receiver. Some types of perceptual amplification that occur between chromatophore layers could evolve through a process analogous, although not identical, to that described by Hasson (1989, 1990, 1991, 1997).

(4) The new framework that we provide for thinking about colour patch evolution makes a number of testable predictions (too detailed to repeat here). Some of these predictions could potentially be tested by surveying existing literature, but ultimately, new integrative studies of animal colouration are needed.

\section{AGKNOWLEDGEMENTS}

We are grateful to C. N. Anderson, D. T. Blumstein, P. Nonacs, D. M. Shier and one anonymous referee for comments on earlier versions of this manuscript. We thank A. Shirvanian for help with Fig. 1 and S. Lees for editorial assistance. This research was supported by a National Science Foundation grant (IBN 0130893) to G.F.G. and G.R.K.

\section{REFERENGES}

Andersson, M. (1994). Sexual Selection. Princeton University Press, Princeton.

Andersson, M. \& Iwasa, Y. (1996). Sexual Selection. Trends in Ecology E Evolution 11, 53-58.

Andersson, S., Pryke, S. R., Ornborg, J., Lawes, M.J. \& Andersson, M. (2002). Multiple receivers, multiple ornaments, and a trade-off between agonistic and epigamic signaling in a widowbird. The American Naturalist 160, 683-691.

Archer, S. N. \& Lythgoe, J. N. (1990). The visual pigment basis for cone polymorphism in the guppy, Poecilia reticulata. Vision Research 30, 225-233.

Badyaev, A. V., Hill, G. E., Dunn, P. O. \& Glen, J. G. (2001). Plumage colour as a composite trait: developmental and functional integration of sexual ornamentation. The American Naturalist 158, 221-235.

Bagnara, J. T. (1966). Cytology and cytophysiology of nonmelanophore pigment cells. International Review of Cytology 20, 173-205. 
Bagnara, J. T. \& Hadley, M. E. (1973). Chromatophores and colour change, the comparative physiology of animal pigmentation. Prentice Hall, Englewood Cliffs.

Bagnara, J. T., Taylor, J. D. \& Hadley, M. E. (1968). The dermal chromatophore unit. Fournal of Cell Biology 38, 67-79.

Bendich, A. (1989). Carotenoids and the immune system. Fournal of Nutrition 119, 112-115.

Berglund, A., Bisazza, A. \& Pilastro, A. (1996). Armaments and ornaments: an evolutionary explanation of traits of dual utility. Biological Journal of the Linnean Society 58, 385-399.

Blount, J. D., Metcalfe, N. B., Birkhead, T. R. \& Surai, P. F. (2003). Carotenoid modulation of immune function and sexual attractiveness in Zebra finches. Science 300, 125-127.

Borgia, G. \& Presgraves, D. C. (1998). Coevolution of elaborate male display traits in the spotted bowerbird: an experimental test of the threat reduction hypothesis. Animal Behaviour 56, 1121-1128.

Brooks, R. \& Couldridge, V. (1999). Multiple sexual ornaments coevolve with multiple mating preferences. The American Naturalist 154, 37-45.

Brush, A. H. (1990). Metabolism of carotenoid pigments in birds. The FASEB fournal 4, 2969-2977.

Burton, G. W. (1989). Antioxidant actions of carotenoids. The Fournal of Nutrition 119, 109-111.

Camplani, A., Saino, N. \& Møller, A. P. (1999). Carotenoids, sexual signals and immune function in barn swallows from Chernobyl. Proceedings of the Royal Society of London Series B 266, 1111-1116.

ChitTKa, L. (1992). The colour hexagon - a chromaticity diagram based on photoreceptor excitations as a generalized representation of colour opponency. Fournal of Comparative Physiology a-Sensory Neural and Behavioural Physiology 170, 533-543.

Chittka, L. (1999). Bees, white flowers, and the colour hexagon - A reassessment? No, not yet: comments on the contribution by Vorobyev et al. Naturwissenschaften 86, 595-597.

Connett, J. E., Kuller, L. H., KJelsberg, M. O., Polk, B. F., Coldins, G., Rider, A. \& Hulley, S. B. (1989). Relationship between carotenoids and cancer. Cancer 64, 126-134.

Grapon de Caprona, M. D. \& Ryan, M.J. (1990). Conspecific mate recognition in swordtails. Xiphophorus nigrensis and X. pygmaeus. (Poeciliidae): olfactory and visual cues. Animal Behaviour 39, 290-296.

Darwin, C. (1871). The Descent of Man, and Selection in Relation to Sex. J. Murray, London.

De Ibarra, N. H., Vorobyev, M., Brandt, R. \& Giurfa, M. (2000). Detection of bright and dim colours by honeybees. The Fournal of Experimental Biology 203, 3289-3298.

Denton, E.J. \& Land, M. F. (1971). Mechanism of reflexion in silvery layers of fish and cephalopods. Proceedings of the Royal Society of London Series A 178, 43-61.

Endler, J. A. (1980). Natural selection on colour patterns in Poecilia reticulata. Evolution 34, 76-91.

ENDLER, J. A. (1991a). Interactions between predators and prey. In Behavioural Ecology, An Evolutionary Approach (eds. J. R. Krebs and N. B. Davies), pp. 169-201. Blackwell, Oxford.

ENDLER, J. A. (1991 b). Variation in the appearance of guppy colour patterns to guppies and their predators under different visual conditions. Vision Research 31, 587-608.

EndLer, J. A. (1992). Signals, signal conditions, and the direction of evolution. The American Naturalist 139, 1-27.

Endler, J. A. (1993). The colour of light in forests and its implications. Ecological Monographs 63, 1-27.
Endler, J. A. \& Houde, A. E. (1995). Geographic variation in female preferences for male traits in Poecilia reticulata. Evolution 49, 456-468.

Endler, J. A. \& Théry, M. (1996). Interacting effects of lek placement, display behaviour, ambient light, and colour patterns in three neotropical forest-dwelling birds. The American Naturalist 148, 421-452.

Faivre, B., Grégoire, A., Préault, M., Cézilly, F. \& Sorci, G. (2003). Immune activation rapidly mirrored in a seconday sexual trait. Science 300, 103.

Fisher, R. A. (1930). The Genetical Theory of Natural Selection. Clarendon Press, Oxford.

Fisher, R. A. (1958). The Genetical Theory of Natural Selection. Dover, New York.

FITZPATRICK, S. (1998a). Birds' tails as signaling devices: markings, shape, length, and feather quality. The American Naturalist 151, $157-173$

FitzPATRICK, S. $(1998 b)$. Colour schemes for birds: structural coloration and signals of quality in feathers. Annales Zoologici Fennici 35, 67-77.

Fleishman, L. J., Bowman, M., Saunders, D., Miller, W. E., RuRY, M. J. \& LoEw, E. R. (1997). The visual ecology of Puerto Rican anoline lizards: habitat light and spectral sensitivity. Fournal of Comparative Physiology a-Sensory Neural and Behavioural Physiology 181, 446-460.

Fleishman, L.J. \& Persons, M. (2001). The influence of stimulus and background colour on signal visibility in the lizard Anolis cristatellus. The fournal of Experimental Biology 204, 1559-1575.

FujII, R. (1993). Cytophysiology of fish chromatophores. International Review of Cytology 143, 191-255.

FujII, R. (2000). The regulation of motile activity in fish chromatophores. Pigment Cell Research 13, 300-319.

FujiI, R., Hayashi, H. \& Toyohara, J. (1991). Analysis of the reflection of light from motile iridophores of the Dark Sleeper, Odontobutis obscura obscura. Zoological Science 8, 461-470.

Goodwin, T. W. (1984). The Biochemistry of the Carotenoids. Chapman $\&$ Hall, London.

GoodwIn, T. W. (1986). Metabolism, nutrition, and function of carotenoids. Annual Review of Nutrition 6, 274-297.

Grafen, A. (1990). Sexual selection unhandicapped by the Fisher process. Fournal of Theoretical Biology 144, 473-516.

Grether, G. F. (1996). Intrasexual competition alone favors a sexually dimorphic ornament in the rubyspot damselfly Hetaerina americana. Evolution 50, 1949-1957.

Grether, G. F. (2000). Carotenoid limitation and mate preference evolution: a test of the indicator hypothesis in guppies (Poecilia reticulata). Evolution 54, 1712-1724.

Grether, G. F., Hudon, J. \& Endler, J. A. (2001a). Carotenoid scarcity, synthetic pteridine pigments and the evolution of sexual coloration in guppies (Poecilia reticulata). Proceedings of the Royal Society of London Series B 268, 1245-1253.

Grether, G. F., Hudon, J. \& Millie, D. F. (1999). Carotenoid limitation of sexual coloration along an environmental gradient in guppies. Proceedings of the Royal Society of London Series B 266, $1317-1322$

Grether, G. F., Kasahara, S., Kolluru, G. R. \& Cooper, E. L. (in press). Sex-specific effects of carotenoid intake on the immunological response to allografts in guppies (Poecilia reticulata). Proceedings of the Royal Society of London Series $B$.

Grether, G. F., Millie, D. F., Bryant, M.J., Reznick, D. N. \& Mayea, W. (2001b). Rain forest canopy cover, resource 
availability, and life history evolution in guppies. Ecology 82, $1546-1559$.

Hankinson, S. J. \& Morris, M. R. (2002). Sexual selection and species recognition in the pygmy swordtail, Xiphophorus pygmaeus: conflicting preferences. Behavioural Ecology and Sociobiology 51, $140-145$.

Hasson, O. (1989). Amplifiers and the handicap principle in sexual selection: a different emphasis. Proceedings of the Royal Society of London Series B 234, 383-406.

Hasson, O. (1990). The role of amplifiers in sexual selection: an intergration of the amplifying and the Fisherian mechanisms. Evolutionary Ecology 4, 277-289.

Hasson, O. (1991). Sexual displays as amplifiers: practical examples with an emphasis on feather decorations. Behavioural Ecology 2, 189-197.

Hasson, O. (1997). Towards a general theory of biological signaling. Fournal of Theoretical Biology 185, 139-156.

Herring, P.J. (1994). Reflective systems in aquatic animals. Comparative Biochememisty and Physiology 109A, 513-546.

HiLl, G. E. (1996). Redness as a measure of the production cost of ornamental coloration. Ethology Ecology \& Evolution 8 , $157-175$.

HiLl, G. E. (1999). Is there an immunological cost to carotenoidbased ornamental coloration? The American Naturalist 154, 589-595.

HiLL, G. E. (2000). Energetic constraints on expression of carotenoid-based plumage coloration. Journal of Avian Biology 31, 559-566.

Holland, B. \& Rice, W. R. (1998). Perspective: chase-away sexual selection: antagonistic seduction versus resistance. Evolution 52, $1-7$.

Houde, A. E. \& Endler, J. A. (1990). Correlated evolution of female mating preferences and male colour patterns in the Guppy Poecilia reticulata. Science 248, 1405-1408.

Houde, A. E. \& Torio, A. J. (1992). Effect of parasitic infection on male colour pattern and female choice in guppies. Behavioural Ecology 3, 346-351.

Hudon, J. (1991). Unusual carotenoid use by the Western tanager (Piranga ludoviciana) and its evolutionary implications. Canadian Fournal of Zoology 69, 2311-2320.

Hudon, J. (1994). Biotechnological applications of research on animal pigmentation. Biotechnology Advances 12, 49-69.

Hudon, J., Grether, G. F. \& Millie, D. F. (in press). Marginal carotenoid differentiation between the sexual and general pigmentation of guppies (Poecilia reticulata) and a possible visual explanation. Physiological and Biochemical Zoology.

Hurst, D. T. (1980). An Introduction to the Chemistry and Biochemistry of Pyrimidines, Purines, and Pteridines. J. Wiley, New York.

Huxley, A. F. (1968). A theoretical treatment of the reflexion of light by multilayer structures. The Fournal of Experimental Biology 48, 227-245.

Inouye, G. Y., Hill, G. E., Stradi, R. D. \& Montgomerie, R. (2001). Carotenoid pigments in male house finch plumage in relation to age, subspecies, and ornamental coloration. The Auk 118, 900-915.

Iwasa, Y. \& Pomiankowski, A. (1994). The evolution of mate preferences for multiple sexual ornaments. Evolution 48 , 853-867.

Jennions, M. D. \& Petrie, M. (1997). Variation in mate choice and mating preferences: a review of causes and consequences. Biological Reviewes of the Cambridge Philosophical Society 72, 283-327.
Jirotkul, M. (1999). Operational sex ratio influences female preference and male-male competition in guppies. Animal Behaviour 58, 287-294.

Johnstone, R. A. (1995). Honest advertisement of multiple qualities using multiple signals. Fournal of Theoretical Biology 177, 87-94.

Johnstone, R. A. (1996). Multiple displays in animal communication: 'backup signals' and 'multiple messages'. Philosophical Transactions of the Royal Society of London B 351, 329-338.

Kayser, H. (1985). Pigments. In Comparative Insect Physiology (eds. G. A. Kerkuts and L. E. Gilbert), pp. 367-415. Academic Press, New York.

Kelber, A., Vorobyev, M. \& Osorio, D. (2003). Animal colour vision - behavioural tests and physiological concepts. Biological Reviews of the Cambridge Philosophical Society 78, 81-118.

Kodric-Brown, A. (1989). Dietary carotenoids and male mating success in the guppy: an environmental component to female choice. Behavioural Ecology and Sociobiology 25, 393-401.

Kodric-Brown, A. (1993). Female choice of multiple male criteria in guppies: interacting effects of dominance, coloration and courtship. Behavioural Ecology and Sociobiology 32, 415-420.

Kodric-Brown, A. \& Brown, J. H. (1984). Truth in advertising: the kinds of traits favored by sexual selection. The American Naturalist 124, 309-323.

Kose, M. \& Møller, A. P. (1999). Sexual selection, feather breakage and parasites: the importance of white spots in the tail of the barn swallow (Hirundo rustica). Behavioural Ecology and Sociobiology 45, 430-436.

Krinsky, N. I. (1989). Carotenoids and cancer in animal models. Fournal of Nutrition 119, 123-126.

Laughlin, S. (1981). Neural principles in the peripheral visusal systems of invertebrates. In Invertebrate Visual Centres and Behaviour (ed. H. J. Autrum), pp. 133-280. Springer, New York.

Leal, M. \& Fleishman, L.J. (2001). Evidence for habitat partitioning based on adaptation to environmental light in a pair of sympatric lizard species. Proceedings of the Royal Society of London Series B 296, 351-359.

Lens, L., Van Dongen, S., Kark, S. \& Matthysen, E. (2002). Fluctuating asymmetry as an indicator of fitness: can we bridge the gap between studies? Biological Reviews of the Cambridge Philosophical Society 77, 27-38.

Losos, J. B. (1985). An experimental demonstration of the species-recognition role of Anolis dewlap color. Copeia 1985, 905-910.

Lozano, G. A. (1994). Carotenoids, parasites, and sexual selection. Oikos 70, 309-311.

Lozano, G. A. (2001). Carotenoids, immunity, and sexual selection: comparing apples and oranges? The American Naturalist 158, 200-203.

Macedonia, J. M. (2001). Habitat light, colour variation, and ultraviolet reflectance in the Grand Cayman anole, Anolis conspersus. Biological fournal of the Linnean Society 73, 299-320.

Macedonia, J. M., Brandt, Y. \& Clark, D. L. (2002). Sexual dichromatism and differential conspicuousness in two populations of the common collared lizard (Crotaphytus collaris) from Utah and New Mexico, USA. Biological Fournal of the Linnean Society 77, 67-85.

Macedonia, J. M., James, S., Wittle, L. W. \& Clark, D. L. (2000). Skin pigments and coloration in the Jamaican radiation of Anolis lizards. Fournal of Herpetology 34, 99-109.

Macedonia, J. M. \& Stamps, J. A. (1994). Species recognition in Anolis grahami (Sauria, Iguanidae) - evidence from responses 
to video playbacks of conspecific and heterospecific displays. Ethology 98, 246-264.

Matsuno, T. (2001). Aquatic animal carotenoids. Fisheries Science 67, 771-783.

Magraw, K. J., Hill, G. E., Stradi, R. \& Parker, R. S. (2001). The influence of carotenoid acquisition and utilization on the maintenance of species-typical plumage pigmentation in male American goldfinches (Carduelis tristis) and northern cardinals (Cardinalis cardinalis). Physiological and Biochemical Zoology 74, 843-852.

McGraw, K.J., Mackillop, E. A., Dale, J. \& Hauber, M. E. (2002). Different colors reveal different information: how nutritional stress affects the expression of melanin- and structurally based ornamental plumage. The Fournal of Experimental Biology 205, 3747-3755.

Møller, A. P., Biard, G., Blount, J. D., Houston, D. G., Ninni, P., Saino, N. \& Surai, P. F. (2000). Carotenoid-dependent signals: indicators of foraging efficiency, immunocompetence or detoxification ability? Avian and Poultry Biology Reviews 11, 137-159.

Møller, A. P. \& Pomiankowski, A. (1993). Why have birds got multiple sexual ornaments? Behavioural Ecology and Sociobiology 32, 167-176.

Møller, A. P. \& Thornhill, R. (1998). Bilateral symmetry and sexual selection: a meta-analysis. American Naturalist 151, 174-192.

Morrison, R. L. (1995). A transmission electron microscope (TEM) method for determining structural colors reflected by lizard iridophores. Pigment Cell Research 8, 28-36.

Morrison, R. L., Rand, M. S. \& Frost-Mason, S. K. (1995). Cellular basis of colour differences in three morphs of the lizard Sceloporus undulatus erythrocheilus. Copeia 1995, 397-408.

Morrison, R. L., Sherbrooke, W. C. \& Frost-Mason, S. K. (1996). Temperature-sensitive, physiologically active iridophores in the lizard Urosaurus ornatus: an ultrastructural analysis of colour change. Copeia 4, 804-812.

Nicol, J. A. C. \& Somiya, H. (1989). The Eyes of Fishes. Oxford University Press, Oxford.

Nielsen, H. I. (1978). Ultrastructural changes in the dermal chromatophore unit of Hyla arborea during colour change. Cell Tissue Research 194, 405-408.

Nielsen, H. I. \& Dyck, J. (1978). Adaptation of the tree frog, Hyla cinerea, to colored backgrounds, and the role of the three chromatophore types. Fournal of Experimental Zoology 205, 79-94.

Овіка, M. (1988). Ultrastructure and physiological response of leucophores of the medaka Oryzias latipes. Zoological Science 5, 311-321.

Oliphant, L. W. \& Hudon, J. (1993). Pteridines as reflecting pigments and components of reflecting organelles in vertebrates. Pigment Cell Research 6, 205-208.

Olson, V. A. \& Owens, I. P. F. (1998). Costly sexual signals: are carotenoids rare, risky or required? Trends in Ecology and Evolution 13, 510-514

Oshima, N. (2001). Direct reception of light by chromatophores of lower vertebrates. Pigment Cell Research 14, 312-319.

PainTER, K.J. (2000). Models for pigment pattern formation in the skin of fishes. Mathematical Models for Biological Pattern Formation 121, 59-82.

Palmer, A. R. (1999). Detecting publication bias in meta-analyses: a case study of fluctuating asymmetry and sexual selection. The American Naturalist 154, 220-233.
Palmer, A. R. (2000). Quasireplication and the contract of error: lessons from sex ratios, heritabilities and fluctuating asymmetry. Annual Review of Ecology and Systematics 31, 441-480.

PArsons, P. A. (1990). Fluctuating asymmetry: an epigenetic measure of stress. Biological Reviews of the Cambridge Philosophical Society 65, 131-145.

Persons, M. H., Fleishman, L. J., Frye, M. A. \& Stimphil, M. E. (1999). Sensory response patterns and the evolution of visual signal design in anoline lizards. Fournal of Comparative Physiology A Sensory Neural and Behavioural Physiology 184, 585-607.

Pfennig, K. S. (1998). The evolution of mate choice and the potential for conflict between species and mate-quality recognition. Proceedings of the Royal Society of London Series B $\mathbf{2 6 5}$, 1743-1748.

Pomiankowski, A. \& Iwasa, Y. (1993). Evolution of multiple sexual preferences by Fisher's runaway process of sexual selection. Proceedings of the Royal Society of London Series B 253, 173-181.

Pryke, S. R., Andersson, S. \& Lawes, M. J. (2001). Sexual selection of multiple handicaps in the red-collared widowbird: female choice of tail length but not carotenoid display. Evolution 55, 1452-1463.

Putnam, M. (1992). A review of the nature, function, variability, and supply of pigments in salmonid fish. In Aquaculture and the Environment (eds. N. Depauw and J. Joyce), pp. 245-263. European Aquaculture Society, Gent, Belgium.

Rempeters, G., Henze, M. \& Anders, F. (1981). Carotenoids and pteridines in the skin of interspecific hybrids of Xiphophorus. Comparative Biochemistry and Physiology 69B, 91-98.

Rodieck, R. W. (1973). The Vertebrate Retina-Priniciples of Structure and Function. Freeman and Company, San Francisco.

Rosenthal, G. G. \& Servedio, M. R. (1999). Chase-away sexual selection: resistance to "resistance". Evolution 53, 296-299.

Rowe, C. (1999). Receiver psychology and the evolution of multicomponent signals. Animal Behaviour 58, 921-931.

Rowe, C. \& Guilford, T. (1999). The evolution of multimodal warning displays. Evolutionary Ecology 13, 655-671.

Ryan, M.J. (1990). Sexual selection, sensory systems, and sensory exploitation. In Oxford Surveys in Evolutionary Biology (eds. D. Futuyma and J. Antonovics), pp. 157-195. Oxford University Press, Oxford.

Ryan, M. J., Fox, J. H., Wilczynski, W. \& Rand, A. S. (1990). Sexual selection for sensory exploitation in the frog Physalaemus pustlosus. Nature 343, 66-67.

Ryan, M. J. \& Keddy-Hector, A. (1992). Directional patterns of female mate choice and the role of sensory biases. The American Naturalist 139, S4-S35.

Schluter, D. \& Price, T. (1993). Honesty, perception and population divergence in sexually selected traits. Proceedings of the Royal Society of London Series B 253, 117-122.

Stavenga, D. G., Smits, R. P. \& Hoenders, B. J. (1993). Simple exponential functions describing the absorbance bands of visual pigment spectra. Vision Research 33, 1011-1017.

Stradi, R., Pini, E. \& Celentano, G. (2001). Carotenoids in bird plumage: the complement of red pigments in the plumage of wild and captive bullfinch (Pyrrhula pyrrhula). Comparative Biochemistry and Physiology Part B 128, 529-535.

Sullivan, M. S. (1994). Mate choice as an information gathering process under time constraint: implications for behaviour and signal design. Animal Behaviour 47, 141-151.

TAKeuchi, I. K. (1976). Electron microscopy of two types of reflecting chromatophores (iridophores and leucophores) in guppy, Lebistes reticulatus Peters. Cell Tissue Research 173, 17-27. 
Taylor, J. D. \& Hadley, M. E. (1970). Chromatophores and colour change in the lizard, Anolis carolinensis. Zeitschrift für Zellforschung 104, 282-294.

Thompson, G. W., Hillgarth, N., Leu, M. \& MaClure, H. E. (1997). High parasite load in house finches (Carpodacus mexicanus) is correlated with reduced expression of a sexually selected trait. The American Naturalist 149, 270-294.

Thompson, C. W. \& Moore, M. C. (1991). Throat colour reliably signals status in male tree lizards, Urosaurus ornatus. Animal Behaviour 42, 745-753.

Thornhill, R., Møller, A. P. \& Gangestad, S. W. (1999). The biological significance of fluctuating asymmetry and sexual selection: a reply to Palmer. American Naturalist 154, 234-241.

Thorpe, R. S. (2002). Analysis of colour spectra in comparative evolution studies: molecular phylogeny and habitat adaptation in the St. Vincent anole (Anolis trinitatis). Systematic Biology 51, 554-569.

von Schantz, T., Bensch, S., Grahn, M., Hasselquist, D. \& Wittzell, H. (1999). Good genes, oxidative stress and condition-dependent sexual signals. Proceedings of the Royal Society of London Series B 266, 1-12.

Vorobyev, M. (1999). Evolution of flower colors - a model against experiments: reply to comments by Chittka. Naturwissenschaften 86, 598-600.

Vorobyev, M., Hempel de Ibarra, N., Brandt, R. \& Giurfa, M. (1999). Do "white" and "green" look the same to a bee? Naturwissenschaften 86, 592-594.

Vorobyev, M., Osorio, D., Bennett, A. T. D., Marshall, N. J. \& Cuthill, I. G. (1998). Tetrachromacy, oil droplets and bird plumage colours. Fournal of Comparative Physiology A Sensory Neural and Behavioural Physiology 183, 621-633.

Wedekind, C., Meyer, P., Frischknecht, M., Niggli, U. A. \& Pfander, H. (1998). Different carotenoids and potential information content of red coloration of male three-spined stickleback. Fournal of Chemical Ecology 24, 787-801.

ZAHAVI, A. (1975). Mate selection - a selection for a handicap. Journal of Theoretical Biology 53, 205-214.

Zuk, M., Ligon, J. D. \& Thornhill, R. (1992). Effects of experimental manipulation of male secondary sex characters on female mate preference in jungle fowl. Animal Behaviour 44, 999-1006.

Zuk, M., Thornhill, R., Ligon, J. D., Johnson, K., Austad, S., Ligon, S. H., Thornhill, N. M. \& Costin, C. (1990). The role of male ornaments and courtship behaviour in female mate choice of red jungle fowl. The American Naturalist 136, 459-473.

\section{APPENDIX A: THE GOLOUR POLYHEDRON}

Here we derive the four-cone geometric equivalent of Chittka's three-cone colour hexagon model, which is briefly described in Section IV.7. For a detailed explanation of the model's assumptions and limitations, see Chittka (1992; also see Chittka, 1999; Vorobyev, 1999; and references therein).

The first step is to orient four unit vectors, representing the four cones $(l, m, s$ and $u)$, at equal angles from each other in three-dimensional space. This can be visualized as a 'tripod', as shown in Fig. Al A. Each vector has a magnitude of one unit because cone excitation levels range from 0 to 1 . For convenience, we define vector $\mathbf{1}$ as parallel to the $y$ axis and vectors $\mathbf{1}$ and $\mathbf{m}$ as lying in the $x y$ plane. Therefore, vector $\mathbf{l}$ projects to $y=1$ and vectors $\mathbf{m}, \mathbf{s}$ and $\mathbf{u}$ project to a plane perpendicular to the $y$ axis and parallel to the $x z$ plane. When all cones are stimulated equally, the sum of the vectors should equal zero in all dimensions (objects that appear white, black or grey map to the achromatic centre of the diagram). It follows that vectors $\mathbf{m}$, $\mathbf{s}$ and $\mathbf{u}$ project to $y=-1 / 3$. Thus, it can be inferred that $\beta$, the angle between the vectors, equals $\cos ^{-1}(-1 / 3) \approx$ $109.4712^{\circ}$. Now we can easily determine the $x$ and $z$ coordinates of the endpoints of each vector trigonometrically, as shown in Fig. Al B, and define the four cone unit vectors as follows:

$$
\begin{aligned}
\mathbf{1} & =[0,1,0], \\
\mathbf{m} & =[\sin \alpha,-1 / 3,0], \\
\mathbf{s} & =[-\cos \theta \sin \alpha,-1 / 3, \sin \theta \sin \alpha], \\
\mathbf{u} & =[-\cos \theta \sin \alpha,-1 / 3,-\sin \theta \sin \alpha],
\end{aligned}
$$

where the numbers in brackets are the $x, y$ and $z$ components of the vectors and angles $\alpha$ and $\theta$ are as defined in Fig. A1. This solution can be verified algebraically by showing that the magnitude of each vector is 1 , that the sum of the four vectors is the zero vector $[0,0,0,0]$, and that the dot product of each pair of vectors is $\cos \beta=-1 / 3$ (not shown).

Solving for the $x, y$ and $z$ coordinates of a particular visual stimulus is a two-step procedure. First, the cone unit vectors are multiplied by the corresponding cone excitation values $E_{j}$ (as defined in equation 9) to yield cone excitation vectors:

$$
\begin{aligned}
\mathbf{l}^{\prime} & =E_{l} \mathbf{l}=\left[0 E_{l}, 1 E_{l}, 0 E_{l}\right]=\left[0, E_{l}, 0\right], \\
\mathbf{m}^{\prime} & =E_{m} \mathbf{m}=\left[\sin \alpha E_{m},-1 / 3 E_{m}, 0 E_{m}\right] \\
& =\left[0.943 E_{m},-0.333 E_{m}, 0\right], \\
\mathbf{s}^{\prime} & =E_{s} \mathbf{s}=\left[-\cos \theta \sin \alpha E_{s},-1 / 3 E_{s}, \sin \theta \sin \alpha E_{s}\right] \\
& =\left[-0.471 E_{s},-0.333 E_{s}, 0.816 E_{s}\right],
\end{aligned}
$$

$$
\begin{aligned}
\mathbf{u}^{\prime} & =E_{u} \mathbf{u}=\left[-\cos \theta \sin \alpha E_{u},-1 / 3 E_{u}^{-}, \sin \theta \sin \alpha E_{u}\right] \\
& =\left[-0.471 E_{u},-0.333 E_{u},-0.816 E_{u}\right],
\end{aligned}
$$

Second, the net cone excitation vector, $\boldsymbol{\phi}$, is obtained by vector addition:

$$
\boldsymbol{\phi}=\mathbf{l}^{\prime}+\mathbf{m}^{\prime}+\mathbf{s}^{\prime}+\mathbf{u}^{\prime},
$$

$$
\begin{aligned}
= & {\left[0.943 E_{m}-0.471\left(E_{s}+E_{u}\right),\right.} \\
& \left.E_{l}-0.333\left(E_{m}+E_{s}+E_{u}\right), 0.816\left(E_{s}-E_{u}\right)\right],
\end{aligned}
$$




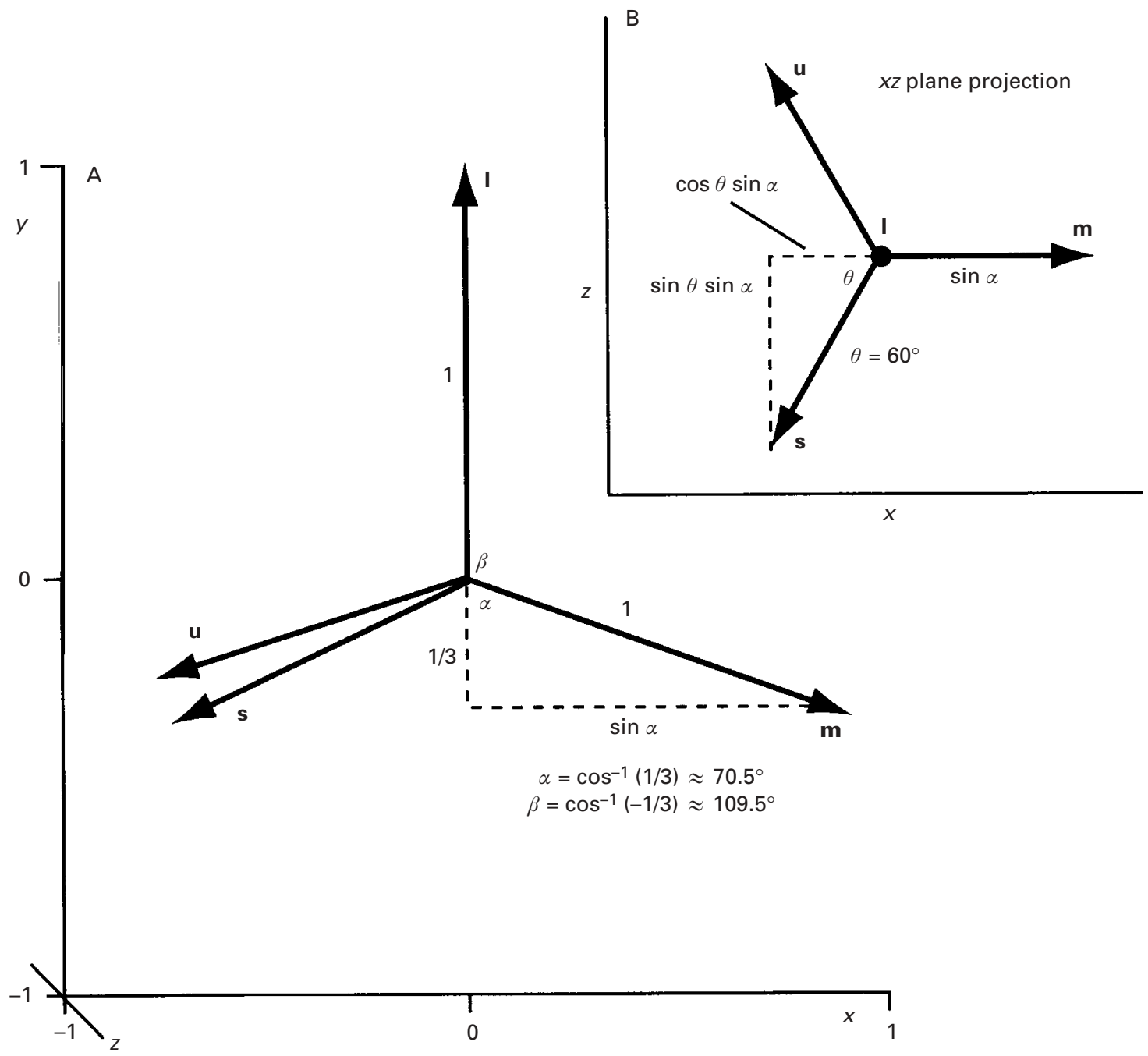

Fig. A1. Vector diagram for deducing the tetrachromatic version of Chittka's (1992) cone-excitation-based colour opponency model. Vectors $\mathbf{1}, \mathbf{m}, \mathbf{s}$ and $\mathbf{u}$, which represent cone classes with different spectral sensitivities, are oriented at equal angles from each other. In A, vectors $\mathbf{l}$ and $\mathbf{m}$ lie in the $x y$ plane (which is tilted slightly relative to the plane of the paper) and vectors $\mathbf{u}$ and $\mathbf{s}$ project below and above the plane of the paper, respectively, in the $z$ dimension. $\mathrm{B}$ is a projection of the vectors onto the $x z$ plane.

$$
=\left[x_{\phi}, y, z_{\phi}\right] .
$$

Vector $\boldsymbol{\phi}$ projects from the origin $(0,0,0)$ to point $\left(x_{\boldsymbol{\phi}}, y_{\boldsymbol{\phi}}, z_{\boldsymbol{\phi}}\right)$. Therefore, $x_{\boldsymbol{\phi}}, y_{\boldsymbol{\phi}}, z_{\boldsymbol{\phi}}$ are the coordinates of the visual stimulus in colour space.

Tetrachromatic colour space encompasses all possible combinations of $x_{\boldsymbol{\phi}}, y_{\boldsymbol{\phi}}, z_{\boldsymbol{\phi}}$. The outer dimensions of this space can be deduced by considering all possible combinations of extreme cone excitation values (the extremes being 0 and 1). There are 16 such combinations, two of which $(0,0,0,0$ and $1,1,1,1)$ map to the geometric centre. From this it can be deduced that tetrachromatic colour space is a polyhedron with 14 vertices, 36 edges and 24 faces (Fig. A2). At four of the vertices, only one cone is maximized (e.g. $1,0,0,0)$; at six vertices exactly two cones are maximized (e.g. 1, 1,0,0); and at the remaining four vertices exactly three cones are maximized (e.g. 1, 1, 1, 0). The six vertices where exactly two cones are maximized are met by four edges and the other eight vertices are met by six edges.

All achromatic stimuli (defined as those for which all $E_{j}$ are equal) map to the centre of the polyhedron. The magnitude of $\boldsymbol{\phi}$, which is calculated from $\left(x_{\boldsymbol{\phi}}{ }^{2}+y_{\boldsymbol{\phi}}{ }^{2}+z_{\boldsymbol{\phi}}{ }^{2}\right)^{0.5}$, provides a measure of chroma or the degree to which a coloured stimulus differs from achromatic stimuli on a 0 to 1 scale.

Equation (A11) provides all the information necessary to calculate tetrachromatic colour distances using equation (19). For example, a bright red object yielding cone 


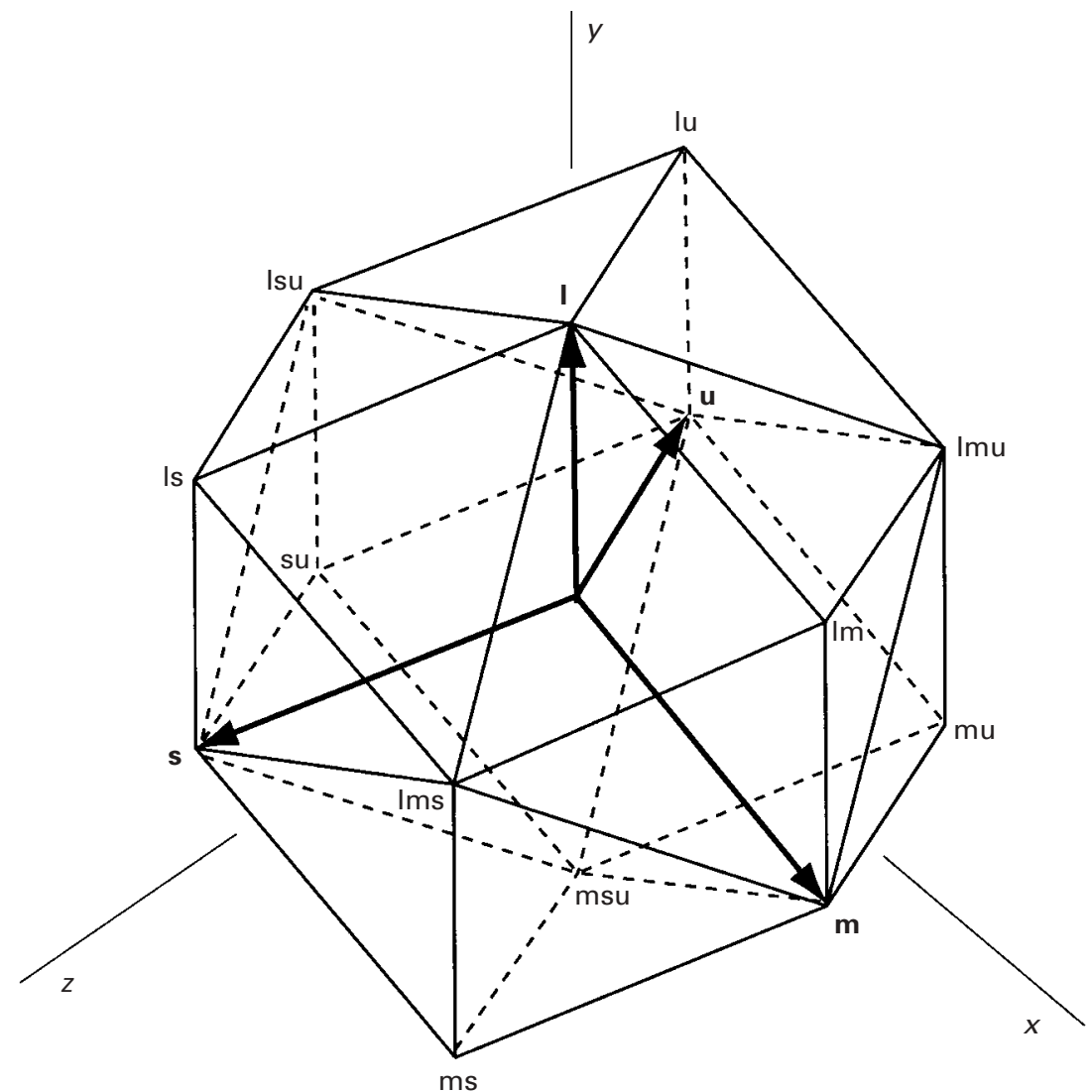

Fig. A2. The colour polyhedron - a generalized, tetrachromatic colour opponency model. All possible excitation levels of four cone types $(l, m, s$ and $u$ ) can be represented in this three-dimensional diagram. The vertices define the theoretical boundaries of colour space and correspond to combinations of maximum and minimum cone excitation vectors, e.g. 'Imu' marks the tip of the net vector $\phi$ when the cone excitation values $E_{l}=1, E_{m}=1, E_{s}=0, E_{u}=1$. Such extreme combinations of excitation levels are impossible in practice, however, because of overlap between the cones in wavelength sensitivity. Visual stimuli that excite all cones equally map to the centre of the diagram and should appear black, grey or white (depending on neural activity in the achromatic channel). Highly chromatic stimuli (e.g. ruby red and cobalt blue) occupy points further from the centre than do weakly chromatic stimuli (e.g. brown and olive green). Euclidean distances between points in colour space provide estimates of perceived colour differences.

excitations of $E_{l}=0.8, E_{m}=0.4, E_{s}=0.1, E_{u}=0.1$ would have the following coordinates and chroma:

$$
\begin{aligned}
& x=0.943(0.4)-0.471(0.1+0.1)=0.283 \\
& y=0.8-0.333(0.4+0.1+0.1)=0.600
\end{aligned}
$$

$$
z=0.816(0.1-0.1)=0
$$

$$
\text { chroma }=\left(0.283^{2}+0.600^{2}+0^{2}\right)^{0.5}=0.663 .
$$

